\title{
Calculation of barriers to proton transfer using multiconfiguration self-consistent-field methods. I. Effects of localization
}

\author{
Karl Luth and Steve Scheiner \\ Department of Chemistry and Biochemistry, Southern Illinois University, Carbondale, Illinois 62901
}

(Received 24 January 1992; accepted 4 August 1992)

\begin{abstract}
The usefulness of multiconfiguration self-consistent-field (MCSCF) calculations in computing correlated proton transfer potentials is investigated for the systems $\mathrm{HF}_{2}^{-}, \mathrm{H}_{7} \mathrm{~N}_{2}^{+}, \mathrm{H}_{3} \mathrm{O}_{2}^{-}$, and $\mathrm{H}_{5} \mathrm{O}_{2}^{+}$. In deciding whether to include particular molecular orbitals, it is important to consider the balance of electron density between the donor and acceptor groups and the interactions that are incorporated in the orbitals. Only orbitals which have the proper symmetry to interact with the transferring hydrogen need be included in the MCSCF active space. Reasonable transfer barriers are obtained when the orbitals are balanced and only interactions relevant to the transfer process are allowed in the MCSCF active space. Equivalent barriers are determined, but the criteria are more easily met, if the canonical molecular orbitals are first subjected to a localization. Only the two localized molecular orbitals that contain the $F$, $\mathrm{N}$, or $\mathrm{O}$ interaction with the transferring hydrogen are needed, which reduces the difficulty of eliminating unproductive interactions. In addition, the localization allows additional virtual orbitals to be included without producing a undesirable correlation.
\end{abstract}

\section{INTRODUCTION}

Because of its fundamental nature and its prevalence in various important chemical and biological reactions, the proton transfer process has been the focus of increasing attention. ${ }^{1-5} \mathrm{~A}$ number of $a b$ initio quantum chemical calculations have been addressed to evaluation of the potential energy surface for the transfer of a proton from one molecule to another across a pre-existing hydrogen bond. Past work has indicated that electron correlation introduces significant perturbations into the Hartree-Fock potentials. $^{6-16}$ The majority of correlated studies of proton transfers have utilized the Møller-Plesset perturbation technique $e^{6-11}$; other methods investigated have included coupled cluster and configuration interaction. ${ }^{12-16}$

Common to these approaches is the assumption that a single electronic configuration can serve as an adequate reference state. While these methods appear to suffice, it would be interesting to examine an alternate approach involving multiple configurations. The central objective of this communication is an analysis of the possibility of using multiconfiguration self-consistent field (MCSCF) methods to calculate proton transfer potentials in an efficient and accurate manner. Such a test has not been carried out as yet in the literature. One strength of this method is its ability to focus in on the correlation associated with particular orbitals. A prime drawback of MCSCF, however, is the necessity to make an arbitrary choice as to which molecular orbitals to include in the expansion. This work hence includes a systematic examination of the effects upon the transfer potential of including various classes and groupings of orbitals in the MCSCF expansion.

A standard Hartree-Fock calculation yields canonical molecular orbitals which are delocalized over the entire complex. These molecular orbitals (MOs) can be transformed into a set of orbitals which are much more concentrated in one region or another of the system by a local- ization procedure. Another question considered is whether such a localization offers a superior framework for the MCSCF machinery in the particular case of the proton transfer process. For example, can the MCSCF procedure be accomplished more efficiently using the localized MOs representing the $\mathrm{X}-\mathrm{H}$ bond and the $\mathrm{Y}$ lone pair, as compared to a larger number of delocalized MOs?

A multiconfiguration method yields a certain fraction of the total correlation energy; the remainder may be recovered by a configuration interaction (CI) approach. There are several starting points for the CI calculations, some of which may be superior to others. In a companion paper, we compare several options. The first is a simple CI expansion using the Hartree-Fock wave function as a single reference. A second uses a MCSCF wave function as a multiconfiguration reference which will also provide information about the viability of single configuration correlation schemes such as Møller-Plesset. In the same spirit of searching for efficiency, we investigate the dual possibilities of performing this preliminary MCSCF in the framework of either canonical or localized MOs.

\section{COMPUTATIONAL DETAILS}

Most of the calculations discussed here were performed using the general atomic and molecular electronic structure system (GAMESS) ${ }^{17}$ The primary focus of this work concerns the MCSCF method, ${ }^{18}$ as implemented in GAMESS. All calculations reported here use the full optimized reaction space (FORS), which includes all possible combinations of electron excitations from the chosen occupied to the chosen virtual orbitals. As a result, the number of configurations included in the calculation rises dramatically with increase in the number of orbitals selected. Our version and implementation of GAMESS allows approximately ten orbitals to be included in the reaction space. Localizations were carried out using the Boys 
criteria, ${ }^{19}$ as implemented in the program. GAUSSIAN-88 (Ref. 20) was used to carry out the Møller-Plesset calculations. The calculations presented employ the split valence 4-31G basis. ${ }^{21}$ Of course, the results with this basis set cannot be taken as definitive values of the transfer barriers but the spirit of this paper is directed more along the lines of examining the merit of each individual computational method rather than determination of a precise barrier of experimental quality. In that vein, the specific basis set choice is less critical than the consistency and reproducibility of the calculated barriers.

The energy barrier for proton transfer is investigated for four symmetric $\mathrm{H}$-bonded complexes, $\mathrm{HF}_{2}^{-}, \mathrm{H}_{7} \mathrm{~N}_{2}^{+}$, $\mathrm{H}_{3} \mathrm{O}_{2}^{-}$, and $\mathrm{H}_{5} \mathrm{O}_{2}^{+}$. The transfer barrier is evaluated as the difference in energy between the midpoint of the transfer and the starting (or ending) point. The rigid molecule approximation is applied so that only the central hydrogen atom is allowed to move during the transfer. All other bond angles and lengths remain constant throughout the transfer. Previous studies have indicated that this is a reasonable approximation for these systems. ${ }^{22-24}$ The midpoint structure is that in which the central hydrogen atom is placed halfway along the $\mathrm{F}-\mathrm{F}, \mathrm{O}-\mathrm{O}$, or $\mathrm{N}-\mathrm{N}$ vector. For the end-point structure, the distance of the bridging hydrogen from the donor atom, along the same axis, is determined by a $6-311 \mathrm{G}^{* *}$ optimization. $\mathbf{H}_{c}$ is used to designate the central hydrogen undergoing the transfer while the terminal hydrogens are denoted $\mathrm{H}_{t}$.

The following geometrical parameters were used to construct the complexes. For $\mathrm{HF}_{2}^{-}$, the F-F distance was set equal to the observed value of $2.28 \AA,{ }^{25}$ resulting in a midpoint $\mathrm{F}-\mathrm{H}_{c}$ distance of $1.14 \AA$. The optimized endpoint structure is somewhat arbitrary for this complex since the potential contains only a single and symmetric minimum. The hydrogen position for the starting point was arbitrarily set slightly closer to one of the fluorines, at a distance of $1.113 \AA$ (the transfer potential is extremely flat for longer distances). The midpoint structure has $D_{\infty h}$ symmetry, while that of the end point is $C_{\infty v}$.

The geometry around each nitrogen atom of $\mathrm{H}_{7} \mathrm{~N}_{2}^{+}$is tetrahedral with $r(\mathrm{NH})$ equal to $1.00 \AA$. The two tetrahedra are staggered with $R(\mathrm{~N}-\mathrm{N})=2.95 \AA$. The $\mathrm{N}-\mathrm{H}_{c}$ distances are 1.475 and $1.043 \AA$ in the mid and end-point structures, respectively. The symmetry of the midpoint is $D_{3 d}$ and the endpoint is $C_{3 v}$.

The length of the bond between the oxygen and each terminal hydrogen $\left(\mathrm{H}_{t}\right)$ in $\mathrm{H}_{3} \mathrm{O}_{2}^{-}$is $0.957 \AA$, and each $\mathrm{O}-\mathrm{H}_{t}$ bond makes an angle of $104.5^{\circ}$ with the $\mathrm{O}-\mathrm{O}$ axis; the two terminal hydrogens are in a trans arrangement with respect to the $\mathrm{O}-\mathrm{O}$ axis. The oxygen atoms are separated by $2.74 \AA$, with $r(\mathrm{OH})$ equal to $0.997 \AA$ in the end-point structure. Even though the midpoint structure is formally of $C_{2 h}$ symmetry, the calculations were limited to $C_{s}$. The end-point structure has $C_{s}$ symmetry.

In each $\mathrm{OH}_{2}$ subunit of $\mathrm{H}_{5} \mathrm{O}_{2}^{+}$, the $\mathrm{O}-\mathrm{H}_{t}$ distances are $0.957 \AA$ and the $\mathrm{H}_{t} \mathrm{OH}_{t}$ angle $104.5^{\circ}$. The $\mathrm{HOH}$ bisectors are disposed $120^{\circ}$ from the $\mathrm{O}-\mathrm{O}$ axis, trans to one another. The $\mathrm{O}-\mathrm{O}$ distance is again $2.74 \AA$. The distance from the nearest oxygen to the central hydrogen is $1.012 \AA$ in the
TABLE I. Proton transfer barriers (kcal/mol).

\begin{tabular}{lccccc}
\hline & Basis set & SCF & MP2 & MP3 & MP4 \\
\hline $\mathrm{HF}_{2}^{-}$ & $4-31 \mathrm{G}$ & -0.059 & -0.105 & -0.092 & -0.077 \\
$\mathrm{H}_{7} \mathrm{~N}_{2}^{+}$ & $4-31 \mathrm{G}$ & 11.15 & 5.61 & 6.94 & 5.96 \\
$\mathrm{H}_{3} \mathrm{O}_{2}^{-}$ & $4-31 \mathrm{G}$ & 6.71 & 0.54 & 2.42 & 1.16 \\
$\mathrm{H}_{5} \mathrm{O}_{2}^{+}$ & $4-31 \mathrm{G}$ & 6.24 & 0.94 & 2.57 & 1.52 \\
$\mathrm{H}_{5} \mathrm{O}_{2}^{+}$ & $6-311 \mathrm{G}^{* *}$ & 10.00 & 4.45 & 6.10 & 4.33 \\
\hline \hline
\end{tabular}

starting structure. The symmetries are $C_{2 h}$ and $C_{s}$ for the mid and end-point structures, respectively. As with $\mathrm{H}_{3} \mathrm{O}_{2}^{-}$, only $C_{s}$ symmetry is explicitly used in the calculations.

As a yardstick of the accuracy of a given calculation, comparisons can be made of the calculated barriers with those found using other methods. Table I reports the barriers computed for each system at the SCF level with the 4-31G basis set as well as the results at various levels of Møller-Plesset perturbation theory. The negative barriers listed for $\mathrm{FHF}^{-}$refer to the greater stability of the midpoint than of the somewhat arbitrary end point since the transfer potential of this system contains a single symmetric well. The trend observed in each case is that MP2 lowers the barrier quite substantially relative to SCF. It is raised a bit by MP3 but lowered again at the MP4 level, all in accord with trends noted previously. ${ }^{16,26,27}$ The data using the larger $6-311 \mathrm{G}^{* *}$ basis set ${ }^{28}$ are included to indicate how changing the size of the basis affects the calculated proton transfer barrier at various levels of correlation. In the following, we will consider the MP4/4-31G results as a sort of benchmark by which to judge the accuracy of the various calculations, also based on 4-31G.

\section{MCSCF CALCULATIONS}

Before discussing the calculations, the SCF orbitals will be summarized. The systems investigated here are isoelectronic, each having 10 occupied orbitals. The MOs in the midpoint geometry represent either symmetric or antisymmetric combinations of the two subunits, with equal weight. The MOs are considerably more localized in the end-point configurations, where each orbital pair consists of two similar orbitals, one located primarily on each subunit. The first two MOs are composed of the $1 s$ orbitals on the first-row atoms, leaving eight valence orbitals. The third and fourth MOs are similar in character except they involve the $2 s$ rather than $1 s$ functions of $\mathrm{F}, \mathrm{O}$, or $\mathrm{N}$, and the terminal hydrogens. The symmetric $\mathrm{MO}$ of this pair also contains a certain contribution from the central hydrogen. The next six MOs incorporate primarily the $p$ orbitals of the first-row atoms and terminal hydrogens where symmetry-allowed.

A prime issue with the MCSCF approach is proper selection of a small number of orbitals to include in the expansion which produce the desired types of correlation. In the case of the proton transfer process, the focus of interest is the $\mathrm{X}-\mathrm{H}_{c}$ bond of the proton donor and the pertinent lone pair of the acceptor molecule. In principle, the MCSCF results depend upon the number of occupied and virtual orbitals of each symmetry that are included in 
TABLE II. Calculated energies (hartree) using combinations of noninteracting orbitals.

\begin{tabular}{|c|c|c|c|c|}
\hline & $\begin{array}{l}\text { Occupied } \\
\text { orbitals }\end{array}$ & $\begin{array}{l}\text { Virtual } \\
\text { orbitals }^{\mathrm{a}}\end{array}$ & $\begin{array}{l}\text { Midpoint } \\
\text { energy }\end{array}$ & $\begin{array}{l}\text { End-point } \\
\text { energy }\end{array}$ \\
\hline \multirow{10}{*}{$\mathrm{HF}_{2}^{-}$} & $1 \pi_{u x} 1 \pi_{u y}$ & $2 \pi_{u x} 2 \pi_{u y}$ & -199.2450872 & -199.2745059 \\
\hline & $1 \pi_{u x} 1 \pi_{u y}$ & $2 \pi_{g x} 2 \pi_{g y}$ & -199.2436681 & -199.2745059 \\
\hline & $1 \pi_{g x} 1 \pi_{g y}$ & $2 \pi_{u x} 2 \pi_{u y}$ & -199.2462302 & -199.2746326 \\
\hline & $1 \pi_{g x} 1 \pi_{g y}$ & $2 \pi_{g x} 2 \pi_{g y}$ & -199.2445013 & -199.2746326 \\
\hline & $1 \pi_{u x} 1 \pi_{u y}$ & $2 \pi_{u x} 2 \pi_{k y} 2 \pi_{g x} 2 \pi_{g y}$ & -199.2526838 & -199.2745065 \\
\hline & $1 \pi_{g x} 1 \pi_{g y}$ & $2 \pi_{u x} 2 \pi_{u y} 2 \pi_{g x} 2 \pi_{g y}$ & -199.2545548 & -199.2746333 \\
\hline & $1 \pi_{u x} 1 \pi_{u y} 1 \pi_{g x} 1 \pi_{g y}$ & $2 \pi_{u x} 2 \pi_{u y}$ & -199.2548402 & -199.2745062 \\
\hline & $1 \pi_{u x} 1 \pi_{\mu y} 1 \pi_{g x} 1 \pi_{g y}$ & $2 \pi_{g x} 2 \pi_{g y}$ & -199.2519336 & -199.2746330 \\
\hline & $1 \pi_{u x} 1 \pi_{u y} 1 \pi_{g x} 1 \pi_{g y}$ & $2 \pi_{u x} 2 \pi_{u y} 2 \pi_{g x} 2 \pi_{g y}$ & -199.3143024 & -199.3142051 \\
\hline & $1 \pi_{u x} 1 \pi_{u y} 1 \pi_{g x} 1 \pi_{g y} 3 \sigma_{g} 3 \sigma_{u}$ & $2 \pi_{u x} 2 \pi_{u y} 2 \pi_{g x} 2 \pi_{g y}$ & -199.3158757 & -199.3157782 \\
\hline \multirow[t]{3}{*}{$\mathrm{H}_{7} \mathrm{~N}_{2}^{+}$} & $1 e_{u x} l e_{u y}$ & $2 e_{u x} 2 e_{u y}$ & -112.6004555 & -112.6433702 \\
\hline & $1 e_{u x} l e_{u y} l e_{g x} l e_{g y}$ & $2 e_{u x} 2 e_{u y} 2 e_{g x} 2 e_{g y}$ & -112.6583554 & -112.6759474 \\
\hline & $1 e_{u x} 1 e_{u y} l e_{g x} 1 e_{g y} 3 a_{2 u} 3 a_{1 g}$ & $2 e_{u x} 2 e_{u y} 2 e_{g x} 2 e_{g y}^{\infty}$ & -112.6611925 & -112.6788464 \\
\hline & $l e_{u x} l e_{u y} l e_{g x} l e_{g y}$ & $e_{u x} 2 e_{u y} 2 e_{g x} 2 e_{g y} 4 a_{2 u} 4 a_{1 g}$ & -112.6692319 & -112.6868471 \\
\hline \multirow[t]{4}{*}{$\mathrm{H}_{3} \mathrm{O}_{2}^{-}$} & $1 a^{\prime \prime} 2 a^{\prime \prime}$ & $3 a^{\prime \prime}$ & -151.1913570 & -151.2080223 \\
\hline & $1 a^{\prime \prime} 2 a^{\prime \prime}$ & $4 a^{\prime \prime}$ & -151.1908534 & -151.2083445 \\
\hline & $1 a^{\prime \prime} 2 a^{\prime \prime}$ & $3 a^{\prime \prime} 4 a^{\prime \prime}$ & -151.2083676 & -151.2189976 \\
\hline & $1 a^{\prime \prime} 2 a^{\prime \prime}$ & $9 a^{\prime} 10 a^{\prime} 11 a^{\prime} 12 a^{\prime} 13 a^{\prime} 14 a^{\prime}$ & -151.1894968 & -151.2003662 \\
\hline \multirow[t]{7}{*}{$\mathrm{H}_{5} \mathrm{O}_{2}^{+}$} & $1 a^{\prime \prime} 2 a^{\prime \prime}$ & $3 a^{\prime \prime}$ & -152.1364188 & -152.1547703 \\
\hline & $1 a^{\prime \prime} 2 a^{\prime \prime}$ & $4 a^{\prime \prime}$ & -152.1363666 & -152.1551280 \\
\hline & $1 a^{\prime \prime} 2 a^{\prime \prime}$ & $3 a^{\prime \prime} 4 a^{\prime \prime}$ & -152.1595144 & -152.1693416 \\
\hline & $1 a^{\prime \prime} 2 a^{\prime \prime}$ & $5 a^{\prime \prime} 6 a^{\prime \prime}$ & -152.1595144 & -152.1693416 \\
\hline & $1 a^{\prime \prime} 2 a^{\prime \prime}$ & $3 a^{\prime \prime} 4 a^{\prime \prime} 5 a^{\prime \prime} 6 a^{\prime \prime}$ & -152.1617649 & -152.1715891 \\
\hline & $1 a^{\prime \prime} 2 a^{\prime \prime}$ & $3 a^{\prime \prime} 4 a^{\prime \prime} 9 a^{\prime} 10 a^{\prime}$ & -152.1646760 & -152.1744897 \\
\hline & $1 a^{\prime \prime} 2 a^{\prime \prime}$ & $11 a^{\prime} 13 a^{\prime}$ & -152.1376799 & -152.1475723 \\
\hline
\end{tabular}

Symmetry designations correspond to the midpoint structure.

the active space. However, the situation may be complicated by the presence of additional local minima that can trap the calculation in a different correlated space, producing undesired results. It is therefore necessary to examine the orbitals resulting from each MCSCF calculation.

One might reasonably suppose that only orbitals which belong to a symmetry designation capable of interacting with the central hydrogen need be included in a MCSCF evaluation of the transfer barrier. This assertion was tested by performing three types of calculations. A first group of orbitals to include in the excitation procedure is a partial set of those which cannot interact with $\mathrm{H}_{c}$. A second choice involves a more complete set of noninteracting orbitals, viz., all of the noninteracting occupied orbitals and their corresponding virtual MOs. A third choice builds on the second in that, in addition to a complete sample of noninteracting orbitals, one includes occupied or virtual orbitals that may interact. As an additional test, this group may also include combinations of occupied noninteracting and virtual interacting orbitals (and vice versa).

Since the basis of the central hydrogen consists entirely of $s$ orbitals within the context of the $4-31 \mathrm{G}$ basis set, $\mathrm{H}_{c}$ can participate (in the midpoint geometry) only in MOs which are symmetric with respect to a plane perpendicular to the H-bond axis. Additional orbitals are able to interact in the case of the end point due to its lower symmetry. As both $\mathrm{HF}_{2}^{-}$and $\mathrm{H}_{7} \mathrm{~N}_{2}^{+}$contain an axis of symmetry coincident with the X-H-X axis, only the molecular orbitals consisting primarily of the atomic $\mathrm{F}$ and $\mathrm{N} 2 s$ and $2 p_{z}$ orbitals, of $\sigma$ or $a$-type symmetry, are able to interact with the central hydrogen. The orbitals containing the $F$ and
$\mathrm{N} 2 p_{x}$ and $2 p_{y}$ atomic orbitals are orthogonal to this axis and belong to the $\pi$ or $e$ symmetry designations and hence cannot interact with the central hydrogen. In contrast, the $\mathrm{O}-\mathrm{H}-\mathrm{O}$ axis of $\mathrm{H}_{5} \mathrm{O}_{2}^{+}$and $\mathrm{H}_{3} \mathrm{O}_{2}^{-}$is not a proper rotation axis. There is, however, a symmetry plane which contains the $\mathrm{O}-\mathrm{H}-\mathrm{O}$ line as well as the terminal hydrogens in $\mathrm{H}_{3} \mathrm{O}_{2}^{-}$; it bisects the $\mathrm{H}_{t}-\mathrm{O}-\mathrm{H}_{t}$ angles in $\mathrm{H}_{5} \mathrm{O}_{2}^{+}$. The six $2 s$, $2 p_{x}$, and $2 p_{y}$ orbitals contained in this plane can interact with the central hydrogen, while the two $2 p_{z}$ orbitals perpendicular to this plane cannot. In these systems, it is the $a^{\prime \prime}$ orbitals which are prohibited from interacting with the central hydrogen while interaction with $a^{\prime}$ is allowed.

\section{A. Noninteracting orbitals}

The results of a series of calculations using noninteracting orbitals are listed in Table II. For $\mathrm{HF}_{2}^{-}$, the first four computations reported use one of the two occupied $\pi$ pairs of orbitals along with one of the virtual $\pi$ pairs. Each of the midpoint calculations produces a different energy. Only two different energies arise for the end-point geometry, suggesting there is one local minimum for each pair of occupied MOs. Regardless of the particular combination of orbitals, the transfer barrier is quite high, ranging between 17.8 and $19.4 \mathrm{kcal} / \mathrm{mol}$. This result reflects a strong imbalance since the SCF data indicate a single symmetric minimum in which a central position of the proton is favored. That is, the barrier computed as the energy difference between the two proton positions should yield a negative barrier, a result confirmed by Table I. Rows 5 and 6 of Table II add another pair of virtual $\pi$ MOs to the set while 7 and 8 add a pair of occupied $\pi$ MOs. Either expansion 
results in a small reduction in the barrier to the 12-14 $\mathrm{kcal} / \mathrm{mol}$ range by increasing the midpoint correlation without significantly increasing that for the end point. A much better result is achieved if all four pairs are included, as in the next calculation which produces a negative barrier, equal to the SCF value. Addition of two $\sigma \mathrm{MOs}$ in the next calculation lowers the energies of both structures slightly, but by similar amounts, leaving the barrier little changed.

As in the $\mathrm{HF}_{2}^{-}$case, use of only one pair of occupied and vacant $e$ MOs yields a barrier much too high in $\mathrm{H}_{7} \mathrm{~N}_{2}^{+}$, as may be seen by the first row of that portion of Table II. Expanding the list to all four pairs lowers the barrier to a more realistic result, within $0.1 \mathrm{kcal} / \mathrm{mol}$ of the SCF value, and again, addition of either occupied or virtual $a$-type MOs, which can interact with the central hydrogen, have minimal effect on the barrier.

The situation for the oxygen-containing systems is somewhat different in that there are only two occupied $a^{\prime \prime}$ MOs prohibited by their symmetry from interacting with the central hydrogen. Taking these two and adding one $a^{\prime \prime}$ virtual produces a rather high barrier, as in the first two calculations for $\mathrm{H}_{3} \mathrm{O}_{2}^{-}$and $\mathrm{H}_{5} \mathrm{O}_{2}^{+}$. Adding a second vacant $a^{\prime \prime}$ reduces the barrier to within $0.1 \mathrm{kcal} / \mathrm{mol}$ of the SCF value in either case. This barrier undergoes very little change thereafter whether more vacant $a^{\prime \prime}$ MOs are added or if the vacant list includes $a^{\prime}$ MOs. The differing individual energies for each particular combination indicates there is no local minimum trapping the MCSCF procedure.

The high barriers can be attributed to a preferential stabilization by the MCSCF procedure of the end point of the transfer in comparison to the midpoint. This preference may be associated with the change in character of the individual MOs as the proton is transferred. For instance, the $1 \pi_{u x}$ and $1 \pi_{u y}$ MOs of $\mathrm{HF}_{2}^{-}$distribute themselves evenly among all four atomic $p_{x}$ and $p_{y}$ orbitals in the midpoint structure, while in the end-point structure they are concentrated on the $p$ orbitals of the proton donor atom. Excitation from these MOs will therefore be similarly concentrated on the donor atom in the end-point structure in comparison to the midpoint. However, the $\left(1 \pi_{g x}, 1 \pi_{g y}\right)$ pair undergoes a reverse polarization in which density accumulates on the acceptor rather than the donor. Combination of the latter pair with $\left(1 \pi_{u x}, 1 \pi_{u y}\right)$ can offer a more balanced framework. Indeed, use of both pairs in the excitation list, along with the four corresponding virtual MOs does result in a much lower barrier (see calculation 9), near the SCF value. The same situation applies to $\mathrm{H}_{7} \mathrm{~N}_{2}^{+}$where the $\left(1 e_{u x}, 1 e_{u y}\right)$ pair must be combined with $\left(1 e_{g x}, 1 e_{g y}\right)$ to achieve the necessary balance. Similar conclusions are reached for $\mathrm{H}_{3} \mathrm{O}_{2}^{-}$and $\mathrm{H}_{5} \mathrm{O}_{2}^{+}$where balance requires $1 a^{\prime \prime}$ and $2 a^{\prime \prime}$ as occupied MOs (coupled with appropriate virtual pairs).

In total then, when one utilizes a complete treatment of orbitals that are not symmetrically disposed to interact with the transferring hydrogen, the SCF barrier is obtained, indicating that the hydrogen position is not an important factor in the amount of correlation resulting from these orbitals. As a result, they need not be included in the allowed excitations of these calculations. However, erratic results arise from an incomplete treatment. The barriers are considerably higher than better correlated calculations would indicate and even higher than the uncorrelated SCF results.

\section{B. Interacting orbitals}

We now shift our attention to those orbitals which are allowed by symmetry to interact directly with the central hydrogen. In the case of the linear $(F-H \cdots F)^{-}$system, these are $\sigma$ orbitals. In order to generate a balanced set of MOs for the MCSCF expansion, we list in Table III the atomic populations obtained for individual MOs. For example, the first row of Table III shows that the atomic population of the proton donor $\mathrm{F}$ atom within the $3 \sigma_{g} \mathrm{MO}$ in the end-point configuration of $(\mathrm{F}-\mathrm{H} \cdots \mathrm{F})^{-}$is 0.952 , as compared to only 0.705 on the acceptor atom. On the other hand, the $3 \sigma_{u}$ MO is of opposite polarity, i.e., the acceptor $\mathrm{F}$ atom is more heavily populated than the donor. When summed together, these two MOs lead to very nearly the same total population on the two $\mathrm{F}$ atoms, as may be seen in Table III. Moreover, this total of $1.819-1.837$ is quite close to the population of 1.828 on the $F$ atoms in the midpoint geometry. In addition to insuring that the populations of the donor and acceptor atoms are not changed drastically, it is also important that the same be true of the transferring hydrogen itself. Table III shows that the population of this atom within the $\left(3 \sigma_{g}, 3 \sigma_{u}\right)$ pair is 0.344 in both the end-point and midpoint geometries. The $\left(3 \sigma_{g}, 3 \sigma_{u}\right)$ pair may hence be thought of as "balanced." Augmentation by the $2 \sigma_{g}$ MO destroys this balance but it is regained if the $2 \sigma_{u}$ MO is also included in the set.

Similarly, the $\left(2 a_{1 g}, 2 a_{2 u}\right)$ pair of $\mathrm{H}_{7} \mathrm{~N}_{2}^{+}$balances out the populations in the two $\mathrm{N}$ atoms fairly well; the central proton participates in this pair only to a small degree, with population $<0.04$. The $\left(3 a_{1 g}, 3 a_{2 u}\right)$ pair, on the other hand, contains high participation by this hydrogen $(>0.4)$ although the balance between the $\mathrm{N}$ atoms is not as good in the $\left(3 a_{1,}, 3 a_{2 u}\right)$ pair. Including both pairs leaves the hydrogen well populated and improves the balance between the $\mathrm{N}$ atoms [compared to the $\left(3 a_{1 g}, 3 a_{2 u}\right)$ pair]. Other combinations listed in Table III leave substantially more density on one $\mathrm{N}$ atom or the other.

Due to the lower symmetry of $\mathrm{H}_{3} \mathrm{O}_{2}^{-}$, there are only two occupied orbitals which are prohibited from interacting with $\mathrm{H}_{c}$. Because of their low energy, we exclude the core and the $2 s$-based orbitals, leaving four occupied $a^{\prime}$ MOs. The $5 a^{\prime}$ and $7 a^{\prime}$ MOs are concentrated on the proton donor $O$ atom (in the end-point structure) while the acceptor is represented by $6 a^{\prime}$ and $8 a^{\prime}$. A pretty good balance, in terms of the two oxygen atom populations as well as $\mathrm{H}_{c}$, results when all four MOs are combined. The data listed in Table III for $\mathrm{H}_{5} \mathrm{O}_{2}^{+}$indicate that taken together, the $\left(7 a^{\prime}, 8 a^{\prime}\right)$ pair is capable of balancing the donor and acceptor atoms rather well; however, there is little interaction with the bridging hydrogen within this pair. The balance is less complete for $\left(5 a^{\prime}, 6 a^{\prime}\right)$ which does involve the hydrogen. Unlike $\mathrm{H}_{3} \mathrm{O}_{2}^{-}$, when both pairs are considered the donor and acceptor remain unbalanced with popula- 
TABLE III. Summation of Hartree-Fock atomic populations.

\begin{tabular}{ccccccc}
\hline \hline & Occupied orbitals & $\begin{array}{c}\mathrm{H}_{c} \\
\text { midpoint }\end{array}$ & $\begin{array}{c}\mathrm{H}_{c} \\
\text { end point }\end{array}$ & $\begin{array}{c}\mathrm{X} \\
\text { midpoint }\end{array}$ & $\begin{array}{c}\mathrm{X}_{\text {don }} \\
\text { end point }\end{array}$ & $\begin{array}{c}\mathrm{X}_{\text {acc }} \\
\text { end point }\end{array}$ \\
\hline $\mathrm{HF}_{2}^{-}$ & $3 \sigma_{g}$ & 0.3438 & 0.3426 & 0.8281 & 0.9522 & 0.7053 \\
& $3 \sigma_{u}$ & 0.0000 & 0.0014 & 1.0000 & 0.8672 & 1.1313 \\
& $3 \sigma_{g} 3 \sigma_{u}$ & 0.3438 & 0.3440 & 1.8281 & 1.8194 & 1.8366 \\
& $2 \sigma_{g} 3 \sigma_{g} 3 \sigma_{u}$ & 0.4383 & 0.4353 & 2.7809 & 3.1852 & 2.3794 \\
$\mathrm{H}_{7} \mathrm{~N}_{2}^{+}$ & $2 \sigma_{g} 2 \sigma_{u} 3 \sigma_{g} 3 \sigma_{u}$ & 0.4383 & 0.4388 & 3.7809 & 3.7693 & 3.7919 \\
& $2 a_{18} 2 a_{2 u}$ & 0.0240 & 0.0377 & 1.6856 & 1.6931 & 1.6210 \\
& $3 a_{1 g} 3 a_{2 u}$ & 0.4530 & 0.4389 & 1.6969 & 1.5131 & 1.8585 \\
& $2 a_{1 g} 2 a_{2 u} 3 a_{1 g} 3 a_{2 u}$ & 0.4770 & 0.4766 & 3.3537 & 3.2062 & 3.4795 \\
& $2 a_{1 g} 2 a_{2 u} 3 a_{1 g}$ & 0.4770 & 0.4152 & 2.3885 & 3.1336 & 1.6692 \\
& $2 a_{1 g} 2 a_{2 u} 3 a_{2 u}$ & 0.0240 & 0.0991 & 2.6220 & 1.7657 & 3.4313 \\
$\mathrm{H}_{3} \mathrm{O}_{2}^{-}$ & $2 a_{1 g} 3 a_{1 g} 3 a_{2 u}$ & 0.4770 & 0.4720 & 2.5234 & 3.2028 & 1.8624 \\
& $2 a_{2 k} 3 a_{1 g} 3 a_{2 u}$ & 0.4530 & 0.4435 & 2.5272 & 1.5165 & 3.4756 \\
& $5 a^{\prime}$ & 0.3803 & 0.2989 & 0.6298 & 1.2810 & 0.0326 \\
& $7 a^{\prime}\left(6 a^{\prime}\right)$ & 0.1134 & 0.0837 & 0.8619 & 1.7229 & 0.0028 \\
& $5 a^{\prime} 7 a^{\prime}\left(5 a^{\prime} 6 a^{\prime}\right)$ & 0.4937 & 0.3826 & 1.4917 & 3.0039 & 0.0354 \\
& $6 a^{\prime} 8 a^{\prime}\left(7 a^{\prime} 8 a^{\prime}\right)$ & 0.0000 & 0.0895 & 1.7408 & 0.1473 & 3.2262 \\
$\mathrm{H}_{3} \mathrm{O}_{2}^{+}$ & $5 a^{\prime} 6 a^{\prime} 7 a^{\prime} 8 a^{\prime}$ & 0.4937 & 0.4721 & 3.2325 & 3.1512 & 3.2616 \\
& $5 a^{\prime} 6 a^{\prime}\left(5 a^{\prime} 7 a^{\prime}\right)$ & 0.3670 & 0.3535 & 1.6478 & 1.5251 & 1.7484 \\
& $7 a^{\prime} 8 a^{\prime}\left(6 a^{\prime} 8 a^{\prime}\right)$ & 0.0193 & 0.0431 & 1.9587 & 1.9536 & 1.9588 \\
& $5 a^{\prime} 6 a^{\prime} 7 a^{\prime} 8 a^{\prime}$ & 0.3863 & 0.3966 & 3.6065 & 3.4787 & 3.7072 \\
\hline \hline
\end{tabular}

symmetry designations correspond to the midpoint structure, except those within parentheses which correspond to the end-point structure.

tions of 3.48 and 3.71 , respectively. The imbalance is due to a shift in the $O$ lone pair density as the hydrogen is transferred.

As mentioned above, there are multiple local minima accessible through the MCSCF procedure. The desired minimum contains mainly "proton transfer" correlation attributed to the $\mathrm{X}-\mathrm{H}_{c}$ bond and the acceptor lone pair, resembling a $p_{\sigma}$ orbital, oriented along the $\mathrm{H}$-bond axis. We wish to avoid nonproductive correlation involving, for example, the terminal $\mathrm{H}$ atoms, even though the resulting total energy may in fact be lower in such a case.

Identification of the multiconfigurational HartreeFock (MCHF) natural orbitals ${ }^{29}$ that result from the MCSCF procedure allows one to focus on the orbitals thought to be most pertinent. Terminal hydrogen incorporation is determined by inspection of the coefficients of the active MCHF natural orbitals. $\mathrm{H}_{t}$ interaction is considered significant if any atomic orbital coefficient is $>0.1$. Extraneous lone pair correlation is avoided by minimizing the $p_{\pi}$ character of the orbitals that are included in the MCSCF active space, where $p_{\pi}$ refers to $p$ orbitals that are oriented perpendicular to the H-bond axis. For each of the complexes studied, several combinations of orbitals, which are detailed in Tables IV-VII, are included in the MCSCF active space to determine which produce the desired correlated space.

Table IV lists the total energies of both the end-point and midpoint geometries of $\mathrm{HF}_{2}^{-}$. Also included is the summation of the occupations of the virtual natural orbitals included in the expansion which provides a measure of the amount of correlation resulting from including these virtuals in the expansion.

The first five rows in the table lead to the same energies for both the end-point and midpoint geometries, indicating that each combination of one occupied and one virtual $\sigma_{g}$ MO yields the same local minimum. The character of the active occupied orbital is identical for each calculation; it is a bonding interaction of $\mathrm{H}_{c}$ with the appropriate combination of fluorine $s$ and $p_{z}$ orbitals, referred to as the $p_{\sigma}$. The virtual natural orbital is similar except it is antibonding and has more $\mathrm{H}_{c}$ character. When additional $\sigma_{g}$ virtuals are included, the second NO is similar to the first but with much smaller occupation; hence little additional correlation energy results. Only when orbitals of opposite parity are added does the amount of productive correlation significantly increase due to creating active orbitals which have different character. The additional occupied and virtual natural orbitals have $p_{\sigma}$ character similar to the original, but the relative orientations are such that there is a node at $\mathrm{H}_{c}$. Even though the added orbitals have no $\mathrm{H}_{c}$ character, they are vital to an accurate representation of the proton transfer process.

The energies and resulting proton transfer barriers from the best MCSCF calculations for each of the complexes are given with respect to active space size in Table VIII. The best computation was defined as that which yields the maximum amount of correlation without significant $\mathrm{H}_{t}$ or $p_{\pi}$ character.

The reader should be reminded that since the transfer potential for $\mathrm{HF}_{2}^{-}$is of the symmetric single well type, the true barrier calculated in this way should be negative. When two occupied orbitals are included in the MCSCF active space, a negative barrier consistent with the MP results of Table I is produced. However, when four occupied orbitals are included the barriers are positive and erratic. The difficulty in obtaining a reasonable barrier in this case is due to a large contribution of the symmetric combination of each fluorine's lone pairs in the occupied set. 
TABLE IV. Calculated energies (hartree) and summation of virtual natural orbital occupations for $\mathrm{HF}_{2}^{-}$.

\begin{tabular}{|c|c|c|c|c|c|}
\hline \multirow[b]{2}{*}{ Occupied orbitals ${ }^{\mathrm{a}}$} & \multirow[b]{2}{*}{ Virtual orbitals ${ }^{\mathbf{a}}$} & \multicolumn{2}{|c|}{ Midpoint } & \multicolumn{2}{|c|}{ End point } \\
\hline & & Energy & Sum & Energy & Sum \\
\hline $2 \sigma_{g}$ & $4 \sigma_{g}$ & -199.2447722 & 0.0084 & -199.2526227 & 0.0144 \\
\hline $2 \sigma_{g}$ & $5 \sigma_{g}^{8}$ & -199.2447722 & 0.0084 & -199.2526227 & 0.0144 \\
\hline $3 \sigma_{g}$ & $4 \sigma_{g}^{\circ}$ & -199.2447722 & 0.0084 & -199.2526227 & 0.0144 \\
\hline $3 \sigma_{g}$ & $5 \sigma_{g}^{\circ}$ & -199.2447722 & 0.0084 & -199.2526227 & 0.0144 \\
\hline $3 \sigma_{g}$ & $6 \sigma_{g}^{\circ}$ & -199.2447722 & 0.0084 & -199.2526227 & 0.0144 \\
\hline $3 \sigma_{u}$ & $4 \sigma_{u}$ & -199.2376996 & 0.0013 & -199.2522459 & 0.0143 \\
\hline $2 \sigma_{g}$ & $4 \sigma_{g} 5 \sigma_{g}$ & -199.2454305 & 0.0086 & -199.2532176 & 0.0147 \\
\hline $3 o_{g}^{8}$ & $4 \sigma_{8}^{8} 5 \sigma_{8}^{\circ}$ & -199.2454305 & 0.0086 & -199.2532176 & 0.0147 \\
\hline $3 \sigma_{k}$ & $4 \sigma_{g} 5 \sigma_{g}$ & -199.2484788 & 0.0145 & -199.2527607 & 0.0147 \\
\hline $2 \sigma_{g}$ & $4 \sigma_{g} 5 \sigma_{g} 6 \sigma_{g}$ & -199.2455025 & 0.0087 & -199.2534038 & 0.0148 \\
\hline $3 \sigma_{g}$ & $4 \sigma_{g} 5 \sigma_{g} 6 \sigma_{g}^{\circ}$ & -199.2455025 & 0.0087 & -199.2534038 & 0.0148 \\
\hline $2 \sigma_{g} 3 \sigma_{g}$ & $4 \sigma_{g}$ & -199.2449193 & 0.0085 & -199.2544658 & 0.0167 \\
\hline $2 \sigma_{g} 3 \sigma_{g}$ & $5 \sigma_{g}$ & -199.2449193 & 0.0085 & -199.2544658 & 0.0167 \\
\hline $2 \sigma_{g}^{\circ} 3 \sigma_{g}$ & $6 \sigma_{g}^{\circ}$ & -199.2449193 & 0.0085 & -199.2544658 & 0.0167 \\
\hline $2 \sigma_{g}^{\circ} 3 \sigma_{g}^{\circ}$ & $4 \sigma_{g} \stackrel{5}{5} \sigma_{g}$ & -199.2477944 & 0.0096 & -199.2687122 & 0.0201 \\
\hline $3 \sigma_{g}^{8} 3 \sigma_{u}^{\circ}$ & $4 \sigma_{g} 5 \sigma_{g}$ & -199.2566214 & 0.0188 & -199.2695203 & 0.0275 \\
\hline $3 \sigma_{g}^{\circ} 3 \sigma_{u}$ & $4 \sigma_{g}^{\circ} 4 \sigma_{u}^{\circ}$ & -199.2696379 & 0.0276 & -199.2695203 & 0.0275 \\
\hline $3 \sigma_{8} 3 \sigma_{u}$ & $5 \sigma_{g} 4 \sigma_{u}$ & -199.2696379 & 0.0276 & -199.2695203 & 0.0275 \\
\hline $2 \sigma_{g} 3 \sigma_{g}$ & $4 \sigma_{g} 5 \sigma_{g} 6 \sigma_{g}$ & -199.2492123 & 0.0099 & -199.2700069 & 0.0213 \\
\hline $3 \sigma_{g} 3 \sigma_{u}$ & $4 \sigma_{g} 5 \sigma_{g} 6 \sigma_{g}$ & -199.2569203 & 0.0194 & -199.2720789 & 0.0293 \\
\hline $3 \sigma_{g} 3 \sigma_{u}$ & $4 \sigma_{g}^{\circ} 5 \sigma_{g} 4 \sigma_{u}^{\circ}$ & -199.2722054 & 0.0294 & -199.2720789 & 0.0293 \\
\hline $3 \sigma_{g} 3 \sigma_{k}$ & $4 \sigma_{g} 6 \sigma_{g} 4 \sigma_{u}$ & -199.2722054 & 0.0294 & -199.2720789 & 0.0293 \\
\hline $3 \sigma_{g} 3 \sigma_{u}$ & $4 \sigma_{g} 5 \sigma_{g} 6 \sigma_{g} 4 \sigma_{u}$ & -199.2726318 & 0.0302 & -199.2725029 & 0.0303 \\
\hline $2 \sigma_{g} 3 \sigma_{g} 3 \sigma_{u}$ & $4 \sigma_{g} 5 \sigma_{g}$ & -199.2568610 & 0.0188 & -199.2698214 & 0.0275 \\
\hline $2 \sigma_{g} 3 \sigma_{g} 3 \sigma_{u}$ & $4 \sigma_{g} \stackrel{\circ}{5} \sigma_{g} 6 \sigma_{g}$ & -199.2603966 & 0.0200 & -199.2860169 & 0.0339 \\
\hline $2 \sigma_{g}^{\circ} 3 \sigma_{g}^{\circ} 3 \sigma_{u}$ & $4 \sigma_{g} 5 \sigma_{g} 6 \sigma_{g} 4 \sigma_{u}$ & -199.2783195 & 0.0358 & -199.2890182 & 0.0317 \\
\hline $2 \sigma_{g} 2 \sigma_{u} 3 \sigma_{g} 3 \sigma_{u}$ & $4 \sigma_{g} 5 \sigma_{g}$ & -199.2625744 & 0.0271 & -199.2694236 & 0.0196 \\
\hline $2 \sigma_{g} 2 \sigma_{u} 3 \sigma_{g} 3 \sigma_{u}$ & $4 \sigma_{g} 5 \sigma_{g} 6 \sigma_{g}$ & -199.2655916 & 0.0277 & -199.2863384 & 0.0341 \\
\hline $2 \sigma_{g} 2 \sigma_{u} 3 \sigma_{g} 3 \sigma_{u}$ & $4 \sigma_{g} 5 \sigma_{g} 6 \sigma_{g} 4 \sigma_{u}$ & -199.2880622 & 0.0404 & -199.3026639 & 0.0343 \\
\hline
\end{tabular}

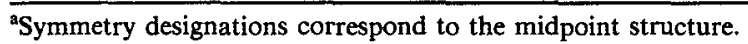

TABLE V. Calculated energies (hartree), summation of virtual natural orbital occupations and largest $\mathrm{H}_{t}$ coefficient for $\mathrm{H}_{7} \mathrm{~N}_{2}^{+}$.

\begin{tabular}{|c|c|c|c|c|c|c|c|}
\hline \multirow[b]{2}{*}{ Occupied orbitals ${ }^{a}$} & \multirow[b]{2}{*}{ Virtual orbitals $^{\mathrm{a}}$} & \multicolumn{3}{|c|}{ Midpoint } & \multicolumn{3}{|c|}{ End point } \\
\hline & & Energy & Sum & $\mathrm{H}_{t}^{\mathrm{b}}$ & Energy & Sum & $\mathbf{H}_{t}^{\mathrm{b}}$ \\
\hline $2 a_{1 g} 2 a_{2 u}$ & $4 a_{1 g} 4 a_{2 u}$ & -112.6253643 & 0.0419 & 0.02 & -112.6367511 & 0.0246 & $0.20(1)$ \\
\hline $3 a_{1 g} 3 a_{2 u}$ & $4 a_{18} 4 a_{2 u}$ & -112.6253643 & 0.0419 & 0.02 & -112.6390208 & 0.0283 & 0.02 \\
\hline $2 a_{1 g} 2 a_{2 u}$ & $4 a_{1 g} 5 a_{1 g} 4 a_{2 k}$ & -112.6285535 & 0.0450 & 0.04 & -112.6389117 & 0.0262 & $0.20(1)$ \\
\hline $3 a_{1 g} 3 a_{2 u}$ & $4 a_{18} 5 a_{1 g} 4 a_{2 u}$ & -112.6285535 & 0.0450 & 0.04 & -112.6415028 & 0.0302 & 0.03 \\
\hline $2 a_{1 g} 2 a_{2 u}$ & $5 a_{1 g} 6 a_{1 g} 5 a_{2 u}$ & -112.6146831 & 0.0299 & $0.13(1)$ & -112.6389117 & 0.0262 & $0.20(1)$ \\
\hline $3 a_{1 g} 3 a_{2 u}$ & $5 a_{1 g} 6 a_{1 g} 5 a_{2 u}$ & -112.6256755 & 0.0423 & $0.37(1)$ & -112.6389117 & 0.0262 & $0.20(1)$ \\
\hline $3 a_{1 g} 3 a_{2 u}$ & $5 a_{1 g} 7 a_{1 g} 6 a_{2 u}$ & -112.6256755 & 0.0423 & $0.37(1)$ & -112.6415028 & 0.0302 & 0.03 \\
\hline $2 a_{1 g} 2 a_{2 u}$ & $4 a_{1 g} 6 a_{1 g} 4 a_{2 u} 5 a_{2 u}$ & -112.6289243 & 0.0458 & 0.09 & -112.6397987 & 0.0268 & $0.62(2)$ \\
\hline $2 a_{1 g} 2 a_{2 u}$ & $4 a_{1 g} 7 a_{1 g} 4 a_{2 u} 6 a_{2 u}$ & -112.6257213 & 0.0424 & $0.46(2)$ & -112.6397966 & 0.0268 & $0.62(2)$ \\
\hline $3 a_{1 g} 3 a_{2 \mu}$ & $4 a_{1 g} 6 a_{1 g} 4 a_{2 u} 5 a_{2 u}$ & -112.6289243 & 0.0458 & 0.09 & -112.6419388 & 0.0305 & $0.30(1)$ \\
\hline $3 a_{1 g} 3 a_{2 u}$ & $4 a_{1 g} 7 a_{1 g} 4 a_{2 u} 6 a_{2 u}$ & -112.6257213 & 0.0424 & $0.46(2)$ & -112.6419409 & 0.0305 & $0.29(1)$ \\
\hline $2 a_{1 g} 3 a_{1 g} 2 a_{2 u}$ & $4 a_{1 g} 4 a_{2 u}$ & -112.6255689 & 0.0422 & 0.02 & -112.6378430 & 0.0263 & $0.19(1)$ \\
\hline $2 a_{1 g} 2 a_{2 u} 3 a_{2 u}$ & $4 a_{1 g}^{\circ} 4 a_{2 u}$ & -112.6254451 & 0.0420 & 0.02 & -112.6378430 & 0.0263 & $0.19(1)$ \\
\hline $2 a_{1 g} 3 a_{1 g} 3 a_{2 u}$ & $4 a_{1 g} 4 a_{2 u}$ & -112.6255689 & 0.0422 & 0.02 & -112.6391721 & 0.0284 & 0.02 \\
\hline $3 a_{1 g} 2 a_{2 u} 3 a_{2 k}$ & $4 a_{1 g} 4 a_{2 u}$ & -112.6254451 & 0.0420 & 0.02 & -112.6380451 & 0.0237 & $0.21(1)$ \\
\hline $2 a_{1 g} 3 a_{1 g} 2 a_{2 u}$ & $4 a_{1 g} 5 a_{1 g} 4 a_{2 u}$ & -112.6299201 & 0.0446 & $0.15(1)$ & -112.6494601 & 0.0330 & $0.23(2)$ \\
\hline $2 a_{1 g} 2 a_{2 u} 3 a_{2 u}$ & $4 a_{1 g} 5 a_{1 g} 4 a_{2 u}$ & -112.6292857 & 0.0401 & $0.17(2)$ & -112.6537430 & 0.0383 & $0.17(2)$ \\
\hline $2 a_{1 g} 3 a_{1 g} 3 a_{2 u}$ & $4 a_{1 g} 5 a_{1 g} 4 a_{2 u}$ & -112.6299200 & 0.0446 & $0.15(1)$ & -112.6494601 & 0.0330 & $0.22(2)$ \\
\hline $3 a_{1 g} 2 a_{2 u} 3 a_{2 u}$ & $4 a_{1 g} 5 a_{1 g} 4 a_{2 u}$ & -112.6308742 & 0.0454 & $0.15(1)$ & -112.6537430 & 0.0383 & $0.17(2)$ \\
\hline $2 a_{1 g} 3 a_{1 g} 2 a_{2 u} 3 a_{2 u}$ & $4 a_{1 g} 4 a_{2 u}$ & -112.6256970 & 0.0422 & 0.02 & -112.6397848 & 0.0263 & $0.19(1)$ \\
\hline $2 a_{1 g} 3 a_{1 g} 2 a_{2 u} 3 a_{2 u}$ & $4 a_{1 g} 5 a_{1 g} 4 a_{2 u}$ & -112.6380384 & 0.0484 & $0.16(2)$ & -112.6506172 & 0.0350 & $0.22(2)$ \\
\hline $2 a_{1 g} 3 a_{1 g} 2 a_{2 u} 3 a_{2 u}$ & $5 a_{1 g} 6 a_{1 g} 5 a_{2 u}$ & -112.6221601 & 0.0338 & $0.16(1)$ & -112.6521845 & 0.0369 & $0.19(2)$ \\
\hline $2 a_{1 g} 3 a_{1 g} 2 a_{2 u} 3 a_{2 u}$ & $5 a_{1 g} 7 a_{1 g} 6 a_{2 u}$ & -112.6359982 & 0.0480 & $0.12(1)$ & -112.6521846 & 0.0369 & $0.19(2)$ \\
\hline $2 a_{1 g} 3 a_{1 g} 2 a_{2 u} 3 a_{2 u}$ & $4 a_{1 g} 6 a_{1 g} 4 a_{2 u} 5 a_{2 u}$ & -112.6418086 & 0.0518 & $0.16(2)$ & -112.6665832 & 0.0469 & $0.18(3)$ \\
\hline $2 a_{1 g} 3 a_{1 g} 2 a_{2 \mu} 3 a_{2 u}$ & $4 a_{1 g} 7 a_{1 g} 4 a_{2 u} 6 a_{2 u}$ & -112.6366302 & 0.0484 & $0.59(2)$ & -112.6665832 & 0.0469 & $0.18(3)$ \\
\hline
\end{tabular}

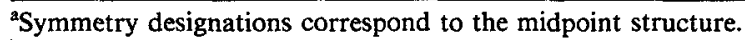

${ }^{b}$ Value in parentheses represents how many of the orbitals have coefficients above 0.10 . 
TABLE VI. Calculated energies (hartree), summation of virtual natural orbital occupations and largest $\mathrm{H}_{t}$ coefficient for $\mathrm{H}_{3} \mathrm{O}_{2}^{-}$.

\begin{tabular}{|c|c|c|c|c|c|c|c|}
\hline \multirow{2}{*}{$\begin{array}{l}\text { Occupied } \\
\text { orbitals" }\end{array}$} & \multirow{2}{*}{$\begin{array}{l}\text { Virtual } \\
\text { orbitals }^{\mathrm{a}}\end{array}$} & \multicolumn{3}{|c|}{ Midpoint } & \multicolumn{3}{|c|}{ End point } \\
\hline & & Energy & Sum & $\mathrm{H}_{t}^{\mathrm{b}}$ & Energy & Sum & $\mathrm{H}_{t}^{\mathrm{b}}$ \\
\hline $5 a^{\prime}$ & $11 a^{\prime}$ & -151.1982243 & 0.0150 & 0.02 & -151.2157954 & 0.0161 & 0.05 \\
\hline $7 a^{\prime}$ & $11 a^{\prime}$ & -151.1982243 & 0.0150 & 0.02 & -151.2157954 & 0.0161 & 0.05 \\
\hline $5 a^{\prime}$ & $11 a^{\prime} 12 a^{\prime}$ & -151.1988443 & 0.0151 & $0.11(1)$ & -151.2220396 & 0.0241 & $1.26(2)$ \\
\hline $7 a^{\prime}$ & $11 a^{\prime} 12 a^{\prime}$ & -151.1988443 & 0.0151 & $0.11(1)$ & -151.2220396 & 0.0241 & $1.26(2)$ \\
\hline $5 a^{\prime} 7 a^{\prime}$ & $11 a^{\prime} 12 a^{\prime}$ & -151.2049839 & 0.0205 & $0.42(1)$ & -151.2478413 & 0.0425 & $0.62(1)$ \\
\hline $6 a^{\prime} 8 a^{\prime}$ & $11 a^{\prime} 12 a^{\prime}$ & -151.2141582 & 0.0340 & $0.48(1)$ & -151.2485842 & 0.0477 & $0.57(1)$ \\
\hline $6 a^{\prime} 8 a^{\prime}$ & $12 a^{\prime} 17 a^{\prime}$ & -151.2070755 & 0.0299 & $0.52(1)$ & -151.2310205 & 0.0315 & $0.11(1)$ \\
\hline $5 a^{\prime} 8 a^{\prime}$ & $11 a^{\prime} 12 a^{\prime}$ & -151.2114634 & 0.0320 & 0.05 & -151.2310205 & 0.0315 & $0.11(1)$ \\
\hline $5 a^{\prime} 8 a^{\prime}$ & $11 a^{\prime} 17 a^{\prime}$ & -151.2247169 & 0.0448 & $0.14(1)$ & -151.2310205 & 0.0315 & $0.11(1)$ \\
\hline $5 a^{\prime} 8 a^{\prime}$ & $12 a^{\prime} 17 a^{\prime}$ & -151.2247169 & 0.0448 & $0.14(1)$ & -151.2310205 & 0.0315 & $0.11(1)$ \\
\hline $5 a^{\prime} 7 a^{\prime}$ & $11 a^{\prime} 12 a^{\prime} 13 a^{\prime}$ & -151.2088423 & 0.0230 & $0.54(2)$ & -151.2524361 & 0.0447 & $1.22(2)$ \\
\hline $5 a^{\prime} 7 a^{\prime}$ & $11 a^{\prime} 12 a^{\prime} 13 a^{\prime} 14 a^{\prime}$ & -151.2108493 & 0.0239 & $0.55(2)$ & -151.2560224 & 0.0473 & $1.22(3)$ \\
\hline $6 a^{\prime} 8 a^{\prime}$ & $9 a^{\prime} 10 a^{\prime} 11 a^{\prime} 12 a^{\prime} 13 a^{\prime} 14 a^{\prime}$ & -151.2244508 & 0.0412 & $0.95(5)$ & -151.2563292 & 0.0560 & $1.31(5)$ \\
\hline $5 a^{\prime} 6 a^{\prime} 7 a^{\prime} 8 a^{\prime}$ & $11 a^{\prime} 12 a^{\prime} 13 a^{\prime} 14 a^{\prime}$ & -151.2763897 & 0.0929 & $0.43(3)$ & -151.2993994 & 0.0905 & $0.62(4)$ \\
\hline $5 a^{\prime} 6 a^{\prime} 7 a^{\prime} 8 a^{\prime}$ & $9 a^{\prime} 10 a^{\prime} 11 a^{\prime} 12 a^{\prime}$ & -151.2763900 & 0.0929 & $0.43(3)$ & -151.2993997 & 0.0905 & $0.62(4)$ \\
\hline $5 a^{\prime} 6 a^{\prime} 7 a^{\prime} 8 a^{\prime}$ & $11 a^{\prime} 12 a^{\prime} 15 a^{\prime} 16 a^{\prime}$ & -151.2763956 & 0.0929 & $0.43(3)$ & -151.2993997 & 0.0905 & $0.62(4)$ \\
\hline $5 a^{\prime} 6 a^{\prime} 7 a^{\prime} 8 a^{\prime}$ & $9 a^{\prime} 10 a^{\prime} 11 a^{\prime} 12 a^{\prime} 13 a^{\prime} 14 a^{\prime}$ & -151.3035186 & 0.1108 & $0.88(4)$ & -151.3091982 & 0.0964 & $1.19(6)$ \\
\hline $5 a^{\prime} 6 a^{\prime} 7 a^{\prime} 8 a^{\prime}$ & $11 a^{\prime} 12 a^{\prime} 13 a^{\prime} 14 a^{\prime} 15 a^{\prime} 16 a^{\prime}$ & -151.3035186 & 0.1108 & $0.88(4)$ & -151.3090187 & 0.0966 & $1.19(6)$ \\
\hline $5 a^{\prime} 6 a^{\prime} 7 a^{\prime} 8 a^{\prime}$ & $9 a^{\prime} 10 a^{\prime} 11 a^{\prime} 12 a^{\prime} 15 a^{\prime} 16 a^{\prime}$ & -151.3035186 & 0.1108 & $0.88(4)$ & -151.3086339 & 0.0965 & $1.19(6)$ \\
\hline
\end{tabular}

Symmetry designations correspond to the midpoint structure.

bValue in parentheses represents how many of the orbitals have coefficients above 0.10 .

TABLE VII. Calculated energies (hartree), summation of virtual natural orbital occupations and largest $\mathrm{H}_{t}$ coefficient for $\mathrm{H}_{5} \mathrm{O}_{2}^{+}$.

\begin{tabular}{|c|c|c|c|c|c|c|c|}
\hline \multirow{2}{*}{$\begin{array}{l}\text { Occupied } \\
\text { orbitals }\end{array}$} & \multirow{2}{*}{$\begin{array}{l}\text { Virtual } \\
\text { orbitals }^{\mathrm{a}}\end{array}$} & \multicolumn{3}{|c|}{ Midpoint } & \multicolumn{3}{|c|}{ End point } \\
\hline & & Energy & Sum & $\mathbf{H}_{t}^{b}$ & Energy & Sum & $\mathrm{H}_{t}^{\mathrm{b}}$ \\
\hline $5 a^{\prime}$ & $11 a^{\prime}$ & -152.1413260 & 0.0133 & 0.02 & -152.1587711 & 0.0156 & 0.02 \\
\hline $5 a^{\prime}$ & $11 a^{\prime} 12 a^{\prime}$ & -152.1418555 & 0.0134 & 0.03 & -152.1600300 & 0.0161 & 0.04 \\
\hline $5 a^{\prime}$ & $9 a^{\prime} 10 a^{\prime} 11 a^{\prime} 3 a^{\prime \prime} 4 a^{\prime \prime}$ & -152.1418888 & 0.0134 & $0.92(3)$ & -152.1605065 & 0.0163 & $0.69(3)$ \\
\hline $5 a^{\prime} 6 a^{\prime}$ & $11 a^{\prime} 12 a^{\prime}$ & -152.1516308 & 0.0240 & 0.03 & -152.1724891 & 0.0278 & 0.04 \\
\hline $5 a^{\prime} 6 a^{\prime}$ & $11 a^{\prime} 13 a^{\prime}$ & -152.1658668 & 0.0374 & 0.05 & -152.1724371 & 0.0272 & $0.33(1)$ \\
\hline $7 a^{\prime} 8 a^{\prime}$ & $9 a^{\prime} 10 a^{\prime}$ & -152.1658668 & 0.0374 & 0.05 & -152.1674515 & 0.0221 & $0.34(1)$ \\
\hline $7 a^{\prime} 8 a^{\prime}$ & $14 a^{\prime} 18 a^{\prime}$ & -152.1362384 & 0.0038 & $0.19(2)$ & -152.1671548 & 0.0227 & $0.33(2)$ \\
\hline $5 a^{\prime} 6 a^{\prime}$ & $11 a^{\prime} 12 a^{\prime} 13 a^{\prime} 15 a^{\prime}$ & -152.1686901 & 0.0404 & $0.16(1)$ & -152.1747244 & 0.0296 & $0.22(1)$ \\
\hline $7 a^{\prime} 8 a^{\prime}$ & $11 a^{\prime} 13 a^{\prime} 14 a^{\prime} 18 a^{\prime}$ & -152.1662426 & 0.0380 & $0.71(2)$ & -152.1875995 & 0.0344 & $0.78(3)$ \\
\hline $5 a^{\prime} 6 a^{\prime} 7 a^{\prime} 8 a^{\prime}$ & $11 a^{\prime} 13 a^{\prime} 14 a^{\prime} 18 a^{\prime}$ & -152.1886999 & 0.0515 & $0.19(2)$ & -152.2255726 & 0.0640 & $0.24(2)$ \\
\hline $5 a^{\prime} 6 a^{\prime} 7 a^{\prime} 8 a^{\prime}$ & $9 a^{\prime} 10 a^{\prime} 11 a^{\prime} 13 a^{\prime} 14 a^{\prime} 18 a^{\prime}$ & -152.2227389 & 0.0758 & $0.66(4)$ & -152.2307686 & 0.0674 & $0.50(3)$ \\
\hline $5 a^{\prime} 6 a^{\prime} 7 a^{\prime} 8 a^{\prime}$ & $11 a^{\prime} 12 a^{\prime} 13 a^{\prime} 14 a^{\prime} 16 a^{\prime} 18 a^{\prime}$ & -152.2261364 & 0.0790 & $0.66(4)$ & -152.2307689 & 0.0674 & $0.50(3)$ \\
\hline
\end{tabular}

"Symmetry designations correspond to the midpoint structure.

balue in parentheses represents how many of the orbitals have coefficients above 0.10 .

TABLE VIII. Calculated energies (hartree) and proton transfer barriers $(\mathrm{kcal} / \mathrm{mol})$ using MCSCF.

\begin{tabular}{lccccc}
\hline \hline & $\begin{array}{c}\text { No. of } \\
\text { occupied }\end{array}$ & $\begin{array}{c}\text { No. of } \\
\text { virtual }\end{array}$ & $\begin{array}{c}\text { Midpoint } \\
\text { energy }\end{array}$ & $\begin{array}{c}\text { End-point } \\
\text { energy }\end{array}$ & $E^{+}$ \\
\hline $\mathrm{HF}_{2}^{-}$ & 2 & 2 & -199.2696379 & -199.2695203 & -0.074 \\
& 2 & 3 & -199.2722054 & -199.2720789 & -0.079 \\
& 2 & 4 & -199.2726318 & -199.2725029 & -0.080 \\
& 4 & 2 & -199.2625744 & -199.2694236 & 4.30 \\
& 4 & 3 & -199.2655916 & -199.2863384 & 13.02 \\
$\mathrm{H}_{7} \mathrm{~N}_{2}^{+}$ & 4 & 4 & -199.2880622 & -199.3026639 & 9.16 \\
& 2 & 2 & -112.6253642 & -112.6390208 & 8.57 \\
& 2 & 3 & -112.6285535 & -112.6415028 & 8.13 \\
$\mathrm{H}_{3} \mathrm{O}_{2}^{-}$ & 2 & 4 & -112.6289239 & -112.6419400 & 8.17 \\
$\mathrm{H}_{5} \mathrm{O}_{2}^{+}$ & 4 & 2 & -112.6256970 & -112.6397848 & 8.84 \\
& 2 & 2 & -151.2247169 & -151.2310205 & 3.96 \\
& 2 & 2 & -152.1658668 & -152.1724891 & 4.16 \\
\hline \hline
\end{tabular}


The relative proportion of donor and acceptor character for the fluorine lone pairs differs for the two structures, resulting in an inconsistent treatment of the interaction when they are included in the active space.

The total energies and virtual NO occupancy summations are presented in Table $\mathrm{V}$ for $\mathrm{H}_{7} \mathrm{~N}_{2}^{+}$along with the largest $\mathrm{H}_{t}$ coefficient of the corresponding orbitals. The first eleven rows contain the results obtained using two occupied orbitals, either $\left(2 a_{1 g}, 2 a_{2 u}\right)$ or $\left(3 a_{1 g}, 3 a_{2 u}\right)$. The MCSCF energy for the midpoint structure does not depend on which occupied pair is used; each produces the same value for a given virtual orbital combination with only one exception. Similar to $\mathrm{HF}_{2}^{-}$, both active natural orbitals, either occupied or virtual, possess $\mathbf{N} p_{\sigma}$ character with one having $\mathrm{H}_{c}$ interaction and the other a node at $\mathrm{H}_{c}$. There is no significant terminal hydrogen involvement in most cases. In some cases where a third virtual MO was added, $\mathrm{H}_{t}$ makes a significant contribution but the occupancy is very low, $<0.0002$.

Unlike the midpoint, the end-point results do depend upon which pair of occupied orbitals is used. The lower energy pair incorporates significant $\mathrm{H}_{t}$ correlation into both the occupied and virtual natural orbitals for any choice of virtuals while the higher energy pair does not. The calculations involving three occupied orbitals are less consistent. While certain combinations lead to identical minima for the midpoint geometry, others do the same in the end point. The inconsistency is due to the occupied orbitals not being balanced between the donor and acceptor atoms, which results in a different correlated space, and thus different natural orbitals, as the central hydrogen moves along the $\mathrm{H}$-bond axis. Although the occupied group containing all four appropriate orbitals is balanced, the results are unpredictable. Only one calculation for either structure avoids terminal hydrogen contributions.

Unlike the $\mathrm{HF}_{2}^{-}$complex where the barriers are highly dependent upon the number of occupied MOs, Table VIII reveals much better consistency for $\mathrm{H}_{7} \mathrm{~N}_{2}^{+}$. The calculated barriers are reasonable, midway between the SCF and MP values. Addition of either occupied or vacant MOs has only a small influence on these quantities. The consistency is due in part to the greater ease with which terminal hydrogen character may be eliminated from the active space in the case of $\mathrm{H}_{7} \mathrm{~N}_{2}^{+}$than $p_{\pi}$ can for $\mathrm{HF}_{2}^{-}$.

It is not sufficient to simply exclude virtuals that have $\mathrm{H}_{t}$ character from the oxygen-containing complexes; one must also examine the relative proportion of $p_{\pi}$ and $p_{\sigma}$. The $p_{\sigma}$ orbitals are central to the transfer but the terminal hydrogens can interact with the oxygen only if $p_{\pi}$ orbitals are included. As a result, significant virtual $p_{\pi}$ character is a mark of unproductive correlation. While only three virtual orbitals meet the above criteria, additional combinations are tested to guarantee productive combinations are not overlooked.

The calculations for $\mathrm{H}_{3} \mathrm{O}_{2}^{-}$reported in the first four rows of Table VI utilize only one occupied orbital, and the results hence favor the end point due to the imbalance between the acceptor and donor. Only two local minima are found for the midpoint and end-point geometries. The next nine calculations describe three different combinations of two occupied orbitals. The $\left(5 a^{\prime}, 7 a^{\prime}\right)$ and $\left(6 a^{\prime}, 8 a^{\prime}\right)$ combinations produce inconsistent results, all of which have a large amount of $\mathrm{H}_{t}$ correlation (coefficients $\geqslant 0.4$ ). The third combination, $\left(5 a^{\prime}, 8 a^{\prime}\right)$, is more consistent in that two of the three energies are equal, and have little terminal hydrogen character in the NOs. The third $\left(5 a^{\prime}, 8 a^{\prime}\right)$ computation has less correlation due to the two virtual orbitals being too similar in character to allow sufficient flexibility.

The last six combinations, which use all four appropriate occupied orbitals, lead to two possible local minima for either the midpoint or end-point geometry. They also result in substantial $\mathrm{H}_{t}$ correlation for any combination of virtuals. No balanced combinations are able to completely eliminate terminal hydrogen correlation. When the occupied combination $\left(5 a^{\prime}, 8 a^{\prime}\right)$ is used with two of the appropriate virtuals, the amount of $\mathbf{H}_{t}$ correlation incorporated is nearly equivalent in both structures. The barrier calculated in this case is $3.96 \mathrm{kcal} / \mathrm{mol}$, as reported in Table VIII, in the regime bordered by the SCF and MP results.

The first three calculations for $\mathrm{H}_{5} \mathrm{O}_{2}^{+}$in Table VII confirm that including one occupied orbital biases the results towards increased end-point correlation. Two combinations of two occupied orbitals are tested. The quality of the results depends on which pair is used. The $\left(5 a^{\prime}, 6 a^{\prime}\right)$ combination yields better results than the $\left(7 a^{\prime}, 8 a^{\prime}\right)$ pair. Correlated space without $\mathrm{H}_{t}$ incorporation is obtained for both midpoint and end point using $\left(5 a^{\prime}, 6 a^{\prime}\right)$ and two virtuals. The midpoint produces two different correlated spaces, one having less correlation again due to the virtual orbitals being too similar. When $\left(5 a^{\prime}, 6 a^{\prime}\right)$ and four virtuals are included in the active space, some terminal hydrogen incorporation results. However, the amount is small enough to consider it an acceptable calculation.

Unlike $\mathrm{H}_{3} \mathrm{O}_{2}^{-}$, either two or four virtual orbitals can be included in the active space without incorporating significant $p_{\pi}$ or $\mathrm{H}_{t}$ character. The barriers calculated are 4.16 and $3.79 \mathrm{kcal} / \mathrm{mol}$ for two and four virtuals, respectively. These values are satisfactory, being slightly larger than those for $\mathrm{H}_{3} \mathrm{O}_{2}^{-}$, as seen for the MP results as well. In addition, as with $\mathrm{H}_{7} \mathrm{~N}_{2}^{+}$the barrier decreases by $\sim 0.4$ $\mathrm{kcal} / \mathrm{mol}$ when flexibility is added by increasing the size of the active space.

Overall, the correlated space, and thus the quality, of a MCSCF calculation is sensitive to the choice of orbitals that are included in the active space. When the correlation is concentrated in orbitals directly involved in the proton transfer process, consistent results are obtained, provided there is a proper balance between the donor and acceptor. Our results indicate that only orbitals which are allowed by symmetry to interact with the transferring hydrogen need be included in the MCSCF active space. However, not all orbitals with the proper symmetry lead to the correct correlated space. Care must be taken to exclude those orbitals, either occupied or virtual, that can introduce interactions other than those pertaining directly to the proton transfer. Using virtual orbitals in addition to one appropriate antibonding counterpart of each occupied orbital lowers the 
TABLE IX. Calculated energies (hartree) and summation of virtual natural orbital occupations using localized occupied orbitals for $\mathrm{HF}_{2}^{-}$.

\begin{tabular}{|c|c|c|c|c|c|}
\hline \multirow{2}{*}{$\begin{array}{l}\text { Occupied } \\
\text { orbitals }\end{array}$} & \multirow[b]{2}{*}{ Virtual orbitals ${ }^{\mathrm{a}}$} & \multicolumn{2}{|c|}{ Midpoint } & \multicolumn{2}{|c|}{ End point } \\
\hline & & Energy & Sum & Energy & Sum \\
\hline $2 \mathrm{~F}-\mathrm{H}_{c}$ & $4 \sigma_{g} 5 \sigma_{g}$ & -199.2566214 & 0.0188 & -199.2695203 & 0.0275 \\
\hline $2 \mathrm{~F}-\mathrm{H}_{c}$ & $6 \sigma_{g} 4 \sigma_{u}$ & -199.2696379 & 0.0276 & -199.2695203 & 0.0275 \\
\hline $2 \mathrm{~F}-\mathrm{H}_{c}$ & $7 \sigma_{g} 5 \sigma_{u}$ & -199.2696379 & 0.0276 & -199.2695203 & 0.0275 \\
\hline $2 \mathrm{~F}-\mathrm{H}_{c}$ & $4 \sigma_{g} 5 \sigma_{g} 6 \sigma_{g}$ & -199.2569203 & 0.0194 & -199.2720789 & 0.0293 \\
\hline $2 \mathrm{~F}-\mathrm{H}_{c}$ & $4 \sigma_{8}^{8} 5 \sigma_{8} 4 \sigma_{u}$ & -199.2722054 & 0.0294 & -199.2720789 & 0.0293 \\
\hline $2 \mathrm{~F}-\mathrm{H}_{c}$ & $4 \sigma_{g} 5 \sigma_{g} 6 \sigma_{g} 4 \sigma_{u}$ & -199.2726318 & 0.0303 & -199.2725030 & 0.0302 \\
\hline $2 \mathrm{~F}-\mathrm{H}_{r}$ & $4 \sigma_{g} 5 \sigma_{g} 7 \sigma_{g} 5 \sigma_{u}$ & -199.2726318 & 0.0303 & -199.2725035 & 0.0302 \\
\hline $2 \mathrm{~F}-\mathrm{H}_{c}$ & $6 \sigma_{g}^{\circ} 7 \sigma_{g} 4 \sigma_{u}^{\circ} 5 \sigma_{u}$ & -199.2725254 & 0.0297 & -199.2725159 & 0.0299 \\
\hline $2 \mathrm{~F}-\mathrm{H}_{6}$ & $4 \sigma_{g} 5 \sigma_{g} 6 \sigma_{g} 7 \sigma_{g} 4 \sigma_{u} 5 \sigma_{u}$ & -199.2733423 & 0.0310 & -199.2732119 & 0.0308 \\
\hline
\end{tabular}

'Symmetry designations correspond to the midpoint structure.

barrier by allowing more correlation to occur without introducing new types of correlation. Finally, it should be noted that it can be difficult to determine in advance of the calculation whether a given set of orbitals will generate the desired correlated space which limits the general usefulness of this approach.

\section{LOCALIZED MCSCF}

The localization procedure changes the character of the occupied orbitals such that each MO corresponds to a distinct bonding or lone pair orbital. For both the end and midpoint geometries of $\mathrm{HF}_{2}^{-}$, localization produces a core orbital, one $\mathrm{F}-\mathrm{H}_{c}$ bonding orbital, and three lone pairs for each $\mathrm{F}$ atom. The four $\mathrm{F}$-centered orbitals are arranged tetrahedrally, relative to the $\mathrm{F}-\mathrm{H}_{c}$ bond lying along the F-F axis. The localized oxygen and nitrogen orbitals in the other complexes adopt the same spatial arrangement. Only the occupied orbitals which consist of the $\mathrm{O}, \mathrm{N}$, or $\mathrm{F}$ interaction with the transferring hydrogen need be included.

The total energies and summation of occupancies using localized occupied orbitals in the MCSCF active space are provided in Tables IX-XII. The localized results for $\mathrm{HF}_{2}^{-}$ are more consistent than those using canonical occupied orbitals. When either two or three virtuals are utilized, the midpoint correlated space depends on their relative parity. If both gerade and ungerade orbitals are included, there is significantly more correlation. This consideration is removed in the endpoint due to loss of the inversion center so any $\sigma$ orbitals provide the desired correlation. With four virtuals there is a small dependence on the number of orbitals of each parity which becomes slightly more pronounced in the midpoint structure.

The total energies and resulting transfer barriers obtained using localized molecular orbitals in the MCSCF active space are listed in Table XIII for each of the complexes. For $\mathrm{HF}_{2}^{-}$, with two vacant orbitals a barrier of

TABLE X. Calculated energies (hartree), summation of virtual natural orbital occupations and largest $\mathrm{H}_{t}$ coefficient using localized occupied orbitals for $\mathrm{H}_{7} \mathrm{~N}_{2}^{+}$.

\begin{tabular}{|c|c|c|c|c|c|c|c|}
\hline \multirow{2}{*}{$\begin{array}{l}\text { Occupied } \\
\text { orbitals }\end{array}$} & \multirow{2}{*}{$\begin{array}{l}\text { Virtual } \\
\text { orbitals }^{\mathrm{a}}\end{array}$} & \multicolumn{3}{|c|}{ Midpoint } & \multicolumn{3}{|c|}{ End point } \\
\hline & & Energy & Sum & $\mathbf{H}_{t}^{b}$ & Energy & Sum & $\mathbf{H}_{t}^{\mathrm{b}}$ \\
\hline $2 \mathrm{~N}-\mathrm{H}_{c}$ & $4 a_{1 g} 4 a_{2 u}$ & -112.6253642 & 0.0419 & 0.02 & -112.6390208 & 0.0283 & 0.01 \\
\hline $2 \mathrm{~N}-\mathrm{H}_{c}$ & $6 a_{1 g} 5 a_{2 u}$ & -112.6253642 & 0.0419 & 0.02 & -112.6390208 & 0.0283 & 0.01 \\
\hline $2 \mathrm{~N}-\mathrm{H}_{c}$ & $7 a_{1 g} 6 a_{2 u}$ & -112.6253642 & 0.0419 & 0.02 & -112.6390208 & 0.0283 & 0.01 \\
\hline $2 \mathrm{~N}-\mathrm{H}_{c}$ & $4 a_{1 g} 5 a_{1 g} 4 a_{2 u}$ & -112.6285535 & 0.0450 & 0.04 & -112.6415028 & 0.0302 & 0.03 \\
\hline $2 \mathrm{~N}-\mathrm{H}_{c}$ & $5 a_{1 g} 6 a_{1 g} 5 a_{2 u}$ & -112.6285535 & 0.0450 & 0.04 & -112.6415028 & 0.0302 & 0.03 \\
\hline $2 \mathrm{~N}-\mathrm{H}_{i}$ & $5 a_{1 g} 7 a_{1 g} 6 a_{2 \mu}$ & -112.6285535 & 0.0450 & 0.04 & -112.6415028 & 0.0302 & 0.03 \\
\hline $2 \mathrm{~N}-\mathrm{H}_{c}$ & $4 a_{1 g} 6 a_{1 g} 4 a_{2 u} 5 a_{2 u}$ & -112.6289760 & 0.0454 & $0.39(1)$ & -112.6419410 & 0.0305 & $0.30(1)$ \\
\hline $2 \mathrm{~N}-\mathrm{H}_{c}$ & $4 a_{1 g} 7 a_{1 g} 4 a_{2 u} 6 a_{2 u}$ & -112.6289767 & 0.0454 & $0.39(1)$ & -112.6419410 & 0.0305 & $0.30(1)$ \\
\hline $2 \mathrm{~N}-\mathrm{H}_{c}$ & $6 a_{1 g} 7 a_{1 g} 5 a_{2 u} 6 a_{2 u}$ & -112.6289769 & 0.0454 & $0.39(1)$ & -112.6419410 & 0.0305 & $0.30(1)$ \\
\hline $2 \mathrm{~N}-\mathrm{H}_{c}$ & $4 a_{1 g} 5 a_{1 g} 6 a_{18} 4 a_{2 u} 5 a_{2 u}$ & -112.6293888 & 0.0462 & $0.38(2)$ & -112.6420992 & 0.0307 & $0.31(2)$ \\
\hline $2 \mathrm{~N}-\mathrm{H}_{c}$ & $4 a_{1 g} 5 a_{1 g} 7 a_{1 g} 4 a_{2 u} 6 a_{2 u}$ & -112.6293888 & 0.0462 & $0.38(2)$ & -112.6420739 & 0.0308 & $0.36(2)$ \\
\hline $2 \mathrm{~N}-\mathrm{H}_{c}$ & $5 a_{1 g} 6 a_{1 g} 7 a_{1 g} 5 a_{2 u} 6 a_{2 u}$ & -112.6293888 & 0.0462 & $0.38(2)$ & -112.6420978 & 0.0307 & $0.31(2)$ \\
\hline $2 \mathrm{~N}-\mathrm{H}_{c}$ & $4 a_{1 g} 5 a_{1 g} 6 a_{1 g} 7 a_{1 g} 4 a_{2 u}$ & -112.6289825 & 0.0459 & $0.20(1)$ & -112.6420988 & 0.0307 & $0.31(2)$ \\
\hline $2 \mathrm{~N}-\mathrm{H}_{c}$ & $4 a_{1 g} 6 a_{1 g} 7 a_{1 g} 4 a_{2 u} 5 a_{2 u} 6 a_{2 u}$ & -112.6294549 & 0.0463 & $0.53(3)$ & -112.6422370 & 0.0310 & $0.42(3)$ \\
\hline $2 \mathrm{~N}-\mathrm{H}_{c}$ & $4 a_{1 g} 5 a_{1 g} 6 a_{1 g} 7 a_{1 g} 4 a_{2 u} 5 a_{2 u} 6 a_{2 u}$ & -112.6296629 & 0.0466 & $0.55(3)$ & -112.6423643 & 0.0313 & $0.57(3)$ \\
\hline
\end{tabular}

asymmetry designations correspond to the midpoint structure.

'Value in parentheses represents how many of the orbitals have coefficients above 0.10 . 
TABLE XI. Calculated energies (hartree), summation of virtual natural orbital occupations and largest $\mathrm{H}_{t}$ coefficient using localized occupied orbitals for $\mathrm{H}_{3} \mathrm{O}_{2}^{-}$.

\begin{tabular}{|c|c|c|c|c|c|c|c|}
\hline \multirow{2}{*}{$\begin{array}{l}\text { Occupied } \\
\text { orbitals }\end{array}$} & \multirow{2}{*}{$\begin{array}{l}\text { Virtual } \\
\text { orbitals }^{\mathbf{a}}\end{array}$} & \multicolumn{3}{|c|}{ Midpoint } & \multicolumn{3}{|c|}{ End point } \\
\hline & & Energy & Sum & $\mathrm{H}_{t}^{\mathrm{b}}$ & Energy & Sum & $\mathrm{H}_{t}^{\mathrm{b}}$ \\
\hline $20-\mathrm{H}_{c}$ & $9 a^{\prime} 10 a^{\prime}$ & -151.2247169 & 0.0448 & $0.14(1)$ & -151.2310205 & 0.0315 & $0.11(1)$ \\
\hline $2 \mathrm{O}-\mathrm{H}_{c}$ & $13 a^{\prime} 14 a^{\prime}$ & -151.2247169 & 0.0448 & $0.14(1)$ & -151.2310205 & 0.0315 & $0.11(1)$ \\
\hline $2 \mathrm{O}-\mathrm{H}_{c}$ & $15 a^{\prime} 16 a^{\prime}$ & -151.2247169 & 0.0448 & $0.14(1)$ & -151.2310205 & 0.0315 & $0.11(1)$ \\
\hline $2 \mathrm{O}-\mathrm{H}_{c}$ & $14 a^{\prime} 16 a^{\prime}$ & -151.2247169 & 0.0448 & $0.14(1)$ & -151.2310205 & 0.0315 & $0.11(1)$ \\
\hline $2 \mathrm{O}-\mathrm{H}_{c}$ & $9 a^{\prime} 10 a^{\prime} 11 a^{\prime} 12 a^{\prime}$ & -151.2283470 & 0.0492 & $0.14(1)$ & -151.2338400 & 0.0339 & $0.78(3)$ \\
\hline $2 \mathrm{O}-\mathrm{H}_{c}$ & $9 a^{\prime} 10 a^{\prime} 14 a^{\prime} 16 a^{\prime}$ & -151.2283470 & 0.0492 & $0.14(1)$ & -151.2338098 & 0.0339 & $0.69(3)$ \\
\hline $20-\mathrm{H}_{c}$ & $11 a^{\prime} 12 a^{\prime} 13 a^{\prime} 14 a^{\prime}$ & -151.2283470 & 0.0492 & $0.14(1)$ & -151.2338481 & 0.0339 & $0.78(3)$ \\
\hline $2 \mathrm{O}-\mathrm{H}_{c}$ & $11 a^{\prime} 12 a^{\prime} 15 a^{\prime} 16 a^{\prime}$ & -151.2283470 & 0.0492 & $0.14(1)$ & -151.2338450 & 0.0339 & $0.11(1)$ \\
\hline $2 \mathrm{O}-\mathrm{H}_{c}$ & $9 a^{\prime} 10 a^{\prime} 13 a^{\prime} 14 a^{\prime}$ & -151.2283054 & 0.0485 & $0.50(2)$ & -151.2338499 & 0.0339 & $0.77(3)$ \\
\hline $2 \mathrm{O}-\mathrm{H}_{c}$ & $9 a^{\prime} 10 a^{\prime} 15 a^{\prime} 16 a^{\prime}$ & -151.2283054 & 0.0485 & $0.50(2)$ & -151.2338504 & 0.0339 & $0.77(3)$ \\
\hline $2 \mathrm{O}-\mathrm{H}_{c}$ & $11 a^{\prime} 12 a^{\prime} 14 a^{\prime} 16 a^{\prime}$ & -151.2120315 & 0.0327 & $0.51(2)$ & -151.2338502 & 0.0339 & $0.77(3)$ \\
\hline $2 \mathrm{O}-\mathrm{H}_{c}$ & $9 a^{\prime} 10 a^{\prime} 11 a^{\prime} 12 a^{\prime} 13 a^{\prime} 14 a^{\prime}$ & -151.2293833 & 0.0516 & $0.54(4)$ & -151.2346575 & 0.0349 & $0.78(5)$ \\
\hline $2 \mathrm{O}-\mathrm{H}_{c}$ & $11 a^{\prime} 12 a^{\prime} 13 a^{\prime} 14 a^{\prime} 15 a^{\prime} 16 a^{\prime}$ & -151.2293833 & 0.0516 & $0.54(4)$ & -151.2346574 & 0.0349 & $0.78(5)$ \\
\hline $20-\mathrm{H}_{c}$ & $9 a^{\prime} 10 a^{\prime} 11 a^{\prime} 12 a^{\prime} 15 a^{\prime} 16 a^{\prime}$ & -151.2293826 & 0.0516 & $0.54(4)$ & -151.2346603 & 0.0350 & $0.78(5)$ \\
\hline $2 \mathrm{O}-\mathrm{H}_{c}$ & $9 a^{\prime} 10 a^{\prime} 11 a^{\prime} 12 a^{\prime} 14 a^{\prime} 16 a^{\prime}$ & -151.2285955 & 0.0493 & $0.54(4)$ & -151.2346629 & 0.0350 & $0.78(5)$ \\
\hline
\end{tabular}

${ }^{2}$ Symmetry designations correspond to the midpoint structure.

${ }^{b}$ Value in parentheses represents how many of the orbitals have coefficients above 0.10 .

TABLE XII. Calculated energies (hartree), summation of virtual natural orbital occupations and largest $\mathrm{H}_{t}$ coefficient using localized occupied orbitals for $\mathrm{H}_{5} \mathrm{O}_{2}^{+}$.

\begin{tabular}{|c|c|c|c|c|c|c|c|}
\hline \multirow{2}{*}{$\begin{array}{l}\text { Occupied } \\
\text { orbitals }\end{array}$} & \multirow{2}{*}{$\begin{array}{l}\text { Virtual } \\
\text { orbitals }^{\mathrm{a}}\end{array}$} & \multicolumn{3}{|c|}{ Midpoint } & \multicolumn{3}{|c|}{ End point } \\
\hline & & Energy & Sum & $\mathbf{H}_{t}^{\mathrm{b}}$ & Energy & Sum & $\mathrm{H}_{t}^{\mathrm{b}}$ \\
\hline $2 \mathrm{O}-\mathrm{H}_{c}$ & $9 a^{\prime} 10 a^{\prime}$ & -152.1685867 & 0.0374 & 0.05 & -152.1724891 & 0.0278 & 0.04 \\
\hline $2 \mathrm{O}-\mathrm{H}_{c}$ & $13 a^{\prime} 14 a^{\prime}$ & -152.1685867 & 0.0374 & 0.05 & -152.1724891 & 0.0278 & 0.04 \\
\hline $2 \mathrm{O}-\mathrm{H}_{c}$ & $11 a^{\prime} 12 a^{\prime}$ & -152.1516302 & 0.0240 & 0.03 & -152.1724891 & 0.0278 & 0.04 \\
\hline $2 \mathrm{O}-\mathrm{H}_{c}$ & $9 a^{\prime} 10 a^{\prime} 11 a^{\prime} 12 a^{\prime}$ & -152.1686901 & 0.0404 & $0.16(1)$ & -152.1747235 & 0.0296 & $0.20(1)$ \\
\hline $2 \mathrm{O}-\mathrm{H}_{c}$ & $9 a^{\prime} 10 a^{\prime} 13 a^{\prime} 14 a^{\prime}$ & -152.1686901 & 0.0404 & $0.16(1)$ & -152.1747244 & 0.0295 & $0.24(1)$ \\
\hline $2 \mathrm{O}-\mathrm{H}_{c}$ & $11 a^{\prime} 12 a^{\prime} 13 a^{\prime} 14 a^{\prime}$ & -152.1520399 & 0.0247 & $0.29(2)$ & -152.1747197 & 0.0296 & $0.16(1)$ \\
\hline $2 \mathrm{O}-\mathrm{H}_{c}$ & $9 a^{\prime} 10 a^{\prime} 11 a^{\prime} 12 a^{\prime} 13 a^{\prime} 14 a^{\prime}$ & -152.1688714 & 0.0405 & $0.52(3)$ & -152.1758242 & 0.0304 & $0.45(3)$ \\
\hline
\end{tabular}

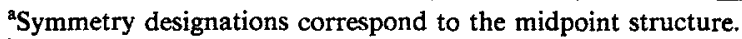

${ }^{b}$ Value in parentheses represents how many of the orbitals have coefficients above 0.10 .

TABLE XIII. Calculated energies (hartree) and proton transfer barriers (kcal/mol) using MCSCF and localized occupied orbitals.

\begin{tabular}{lccccc}
\hline \hline & $\begin{array}{c}\text { No. of } \\
\text { occupied }\end{array}$ & $\begin{array}{c}\text { No. of } \\
\text { virtual }\end{array}$ & $\begin{array}{c}\text { Midpoint } \\
\text { energy }\end{array}$ & $\begin{array}{c}\text { End-point } \\
\text { energy }\end{array}$ & $E^{\dagger}$ \\
\hline $\mathrm{HF}_{2}^{-}$ & 2 & 2 & -199.2696379 & -199.2695203 & -0.0738 \\
& 2 & 3 & -199.2722054 & -199.2720789 & -0.0794 \\
& 2 & 4 & -199.2726318 & -199.2725035 & -0.0805 \\
$\mathrm{H}_{7} \mathrm{~N}_{2}^{+}$ & 2 & 6 & -199.2733423 & -199.2732119 & -0.0818 \\
& 2 & 2 & -112.6253642 & -112.6390208 & 8.57 \\
& 2 & 3 & -112.6285535 & -112.6415038 & 8.13 \\
$\mathrm{H}_{3} \mathrm{O}_{2}^{-}$ & 2 & 4 & -112.6289767 & -112.6419410 & 8.13 \\
& 2 & 2 & -151.2247169 & -151.2310205 & 3.96 \\
$\mathrm{H}_{5} \mathrm{O}_{2}^{+}$ & 2 & 4 & -151.2283470 & -151.2338450 & 3.45 \\
& 2 & 2 & -152.1658668 & -152.1724891 & 4.16 \\
& 2 & 4 & -152.1686901 & -152.1747249 & 3.79 \\
\hline \hline
\end{tabular}


$-0.074 \mathrm{kcal} / \mathrm{mol}$ is obtained. Each additional virtual MO included in the active space changes the barrier only negligibly. The only difference compared to the canonical results is that now six virtuals can be employed. The values are in excellent agreement with the MP barriers in Table I.

For $\mathrm{H}_{7} \mathrm{~N}_{2}^{+}$, as with $\mathrm{HF}_{2}^{-}$, the results are highly consistent, more so than the canonical results. The correlated space is dependent only on the number of orbitals of each symmetry that are included in the virtual active space. It is noteworthy that the addition of virtual orbitals increases the amount of correlation but has a negligible effect on the character of the natural orbitals; it merely adds an additional orbital which has a much smaller occupation. With either two or three virtuals there is no $\mathrm{H}_{t}$ involvement in the correlation, but the addition of a fourth virtual incorporates a very small amount. Addition of further virtuals greatly increases the correlation involving the terminal hydrogens, indicating that only four virtuals are needed to adequately represent the proton transfer process. The energies, correlated space, and barriers for $\mathrm{H}_{7} \mathrm{~N}_{2}^{+}$computed using the two $\mathrm{N}-\mathrm{H}_{c}$ orbitals (Table XIII) are virtually identical to the best values obtained with the canonical orbitals.

For $\mathrm{H}_{3} \mathrm{O}_{2}^{-}$, the data in Table XI reveal that localization does not eliminate $\mathrm{H}_{t}$ correlation. The calculations using two virtuals are consistent and equivalent to the best obtained using canonical orbitals. However, when four virtuals are employed, the results become erratic. Two quite different, but energetically similar, correlated spaces are obtained for each geometry. The more frequent midpoint result is simply an extension of the two-orbital correlated space, similar to the other complexes. The other result has three orbitals with significant $\mathbf{H}_{t}$ incorporation, although the one with the highest occupation is equivalent to the corresponding orbital in the calculations without significant $\mathrm{H}_{t}$ correlation. For the end point, all but one of the calculations produces large amounts of $\mathrm{H}_{t}$ correlation, with only the largest occupied not possessing $\mathrm{H}_{t}$. While using four virtuals is precarious for this complex, the energies are so close even when the $\mathrm{H}_{t}$ correlation is significant, that the barrier listed in Table XIII is reasonably accurate. In addition, the trend of reduction of the calculated barrier for $\mathrm{H}_{7} \mathrm{~N}_{2}^{+}$and $\mathrm{H}_{5} \mathrm{O}_{2}^{+}$by $\sim 0.4 \mathrm{kcal} / \mathrm{mol}$ with increase in the size of the active space holds true for this complex as well.

Table XII presents the energies, largest $\mathrm{H}_{t}$ coefficients, and occupancy summations for $\mathrm{H}_{5} \mathrm{O}_{2}^{+}$. The results are again nearly identical to those obtained using the best combination of canonical orbitals. The main difference is that, once again, certain combinations of the midpoint virtual orbitals do not provide sufficient flexibility and correlation because they too closely resemble one another. As with $\mathrm{H}_{3} \mathrm{O}_{2}^{-}$, the initial $\mathrm{H}_{t}$ correlation is introduced to a very small degree in the fourth natural orbital. By the time the sixth virtual is included, incorporation of terminal hydrogen interaction is excessive.

Overall, the MCSCF results using localized occupied MOs are encouraging. In principle, the final correlated space should not depend on whether canonical or localized MOs are used. Both the barriers and the correlated spaces calculated using localized orbitals are indeed virtually identical to those obtained from the canonical MCSCF calculations, indicating that the best canonical calculations do effectively eliminate $p_{\pi}$ or $\mathbf{H}_{t}$ interaction. Localization simplifies the avoidance of unproductive correlation, allowing the desired correlated space to be reached more consistently. There is an additional advantage of localizing the occupied orbitals; the precise character of the virtual orbitals becomes less crucial so there can be small contributions from $p_{\pi}$ or $\mathrm{H}_{t}$ without creating the additional correlation because those elements do not exist in the localized occupied orbitals. Localization also permits use of additional virtual orbitals, increasing the amount of the desired correlation.

\section{CONCLUSIONS}

The correlated space and thus the quality of a MCSCF calculation is highly sensitive to the choice of orbitals that are included in the active space. It does appear possible to obtain consistent results if one adheres to a set of criteria that include balance of MOs between donor and acceptor atom. Our results indicate that only orbitals which are allowed by symmetry to interact with the transferring hydrogen need be included in the MCSCF active space. In addition, including only virtual orbitals that are devoid of terminal hydrogen or $p_{\pi}$ character produces the best results, avoiding undesired correlation.

Although the MCSCF calculations are usually able to calculate reasonable proton transfer barriers, the consistency of the results is improved by prior localization of the occupied orbitals. The barriers obtained are equivalent to those obtained from canonical MOs, provided that interactions other than those pertaining to the proton transfer process are removed from the MCSCF active space. Localization of the SCF orbitals relieves some of the difficulty in this task by providing orbitals that do not possess $p_{\pi}$ or $\mathrm{H}_{t}$ character. In addition, localization allows virtuals which have some $p_{\pi}$ or $\mathrm{H}_{t}$ contributions to be included in the MCSCF active space without adding in undesired correlation. The additional virtual orbitals result in more productive correlation and improved transfer barriers.

In summary, MCSCF calculations are inferior to other correlated methods such as Møller-Plesset for investigating proton transfer reactions. Selection of appropriate MOs to include in the active space is problematic and requires great care. The prognosis is more favorable if the occupied MOs are localized as the desired correlated space can be reached more consistently. When using Boys localized orbitals, the calculated barriers are similar to those of other correlation methods, albeit slightly higher than the Møller-Plesset values obtained with the same basis set. The barriers obtained are $-0.80 \mathrm{kcal} / \mathrm{mol}$ for $\mathrm{HF}_{2}^{-}, 8.1$ for $\mathrm{H}_{7} \mathrm{~N}_{2}^{+}, 3.4 \mathrm{kcal} / \mathrm{mol}$ for $\mathrm{H}_{3} \mathrm{O}_{2}^{-}$, and 3.8 for $\mathrm{H}_{5} \mathrm{O}_{2}^{+}$.

\section{ACKNOWLEDGMENT}

This work was supported by the National Institutes of Health (GM29391). 
${ }^{1}$ E. F. Caldin and V. Gold, Proton Transfer Reactions (Halsted, New York, 1975).

${ }^{2}$ R. Stewart, The Proton: Applications to Organic Chemistry (Academic, Orlando, 1985).

${ }^{3}$ J. A. Dodd, S. Baer, C. R. Moylan, and J. I. Brauman, J. Am. Chem. Soc. 113, 5942 (1991).

${ }^{4}$ M. Meot-Ner and S. C. Smith, J. Am. Chem. Soc. 113, 862 (1991).

${ }^{5}$ D. W. Firth, K. Beyer, M. A. Dvorak, S. W. Reeve, A. Grushow, and K. R. Leopold, J. Chem. Phys. 94, 1812 (1991).

${ }^{6}$ L. Jaroszewski, B. Lesyng, J. J. Tanner, and J. A. McCammon, Chem. Phys. Lett. 175, 282 (1990).

${ }^{7}$ T. N. Truong and J. A. McCammon, J. Am. Chem. Soc. 113, 7504 (1991).

${ }^{8}$ S. P. Gejji, O. E. Taurian, and S. Lunell, J. Phys. Chem. 94, 4449 (1990).

${ }^{9}$ H. Basch and W. J. Stevens, J. Am. Chem. Soc. 113, 95 (1991).

${ }^{10}$ E. Bosch, J. M. Lluch, and J. Bertran, J. Am. Chem. Soc. 112, 3868 (1990).

${ }^{11}$ M. J. Frisch, A. C. Scheiner, H. F. Schaefer III, and J. S. Binkley, J. Chem. Phys. 82, 4194 (1985).

${ }^{12}$ W. Meyer, W. Jakubetz, and P. Schuster, Chem. Phys. Lett. 21, 97 (1973).

${ }^{13}$ A. Stogård, A. Strich, J. Almlöf, and B. Roos, Chem. Phys. 8, 405 (1975).

${ }^{14}$ B. O. Roos, W. P. Kraemer, and G. H. F. Diercksen, Theor. Chim. Acta 42, 77 (1976).

${ }^{15} \mathrm{G}$. Karlström, B. Jönsson, B. Roos, and H. Wennerström, J. Am. Chem. Soc. 98, 6851 (1976).

${ }^{16}$ Z. Latajka and S. Scheiner, J. Mol. Struct. (Theochem) 234, 373 (1991).

${ }^{17}$ M. Dupuis, D. Spangler, and J. J. Wendoloski, General Atomic and Molecular Electronic Structure System, National Resource for Compu- tational Chemistry (Lawrence Berkeley Laboratory, Berkeley, California, 1980), as modified by M. W. Schmidt, North Dakota State University; S. T. Elbert, Iowa State University.

${ }^{18}$ B. O. Roos, in Methods in computational Molecular Physics, edited by G. H. F. Diercksen and S. Wilson (Reidel, Dordrecht, Netherlands, 1983), p. 161; Olsen, Yeager, Jorgensen, in Advances in Chemical Physics, edited by I. Prigogine and S. A. Rice (Wiley-Interscience, New York, 1983), p. 1; H.-J. Werner and R. Shepard, in Advances in Chemical Physics, edited by K. P. Lawley (Wiley-Interscience, New York, 1987), pp. 1 and 63.

${ }^{19}$ S. F. Boys, Rev. Mad. Phys. 32, 296 (1960).

${ }^{20}$ M. J. Frisch, M. Head-Gordon, H. B. Schlegel, K. Raghavachari, J. S. Binkley, C. Gonzalez, D. J. Defrees, D. J. Fox, R. A. Whiteside, R. Seeger, C. F. Melius, J. Baker, R. Martin, L. R. Kahn, J. J. P. Stewart, E. M. Fluder, S. Topiol, and J. A. Pople, Gaussian, Inc., Pittsburg, PA, 1988.

${ }^{21}$ R. Dirchfield, W. J. Hehre, and J. A. Pople, J. Chem. Phys. 54, 724 (1971); W. J. Hehre, R. Ditchfield, and J. A. Pople, ibid. 56, 2257 (1972); J. B. Collins, P. V. R. Schleyer, J. S. Binkley, and J. A. Pople, J. Phys. Chem. 86, 1529 (1982).

${ }^{22}$ S. Scheiner, Acc. Chem. Res. 18, 174 (1985).

${ }^{23}$ S. Scheiner, J. Am. Chem. Soc. 103, 315 (1981).

${ }^{24}$ S. Scheiner, J. Phys. Chem. 86, 376 (1982).

${ }^{25}$ K. Kawaguchi and E. Hirota, J. Chem. Phys. 84, 2953 (1986), and references therein.

${ }^{26}$ S. Scheiner, M. M. Szczesniak, and L. D. Bigham, Int. J. Quantum Chem. 23, 739 (1983).

${ }^{27}$ M. M. Szczesniak and S. Scheiner, J. Chem. Phys. 77, 4586 (1982).

${ }^{28}$ R. Krishnan, J. S. Binkley, R. Seeger, and J. A. Pople, J. Chem. Phys. 72, $650(1980)$.

${ }^{29}$ R. S. Grev and H. F. Schaefer III, J. Chem. Phys. 96, 6850 (1992). 